\title{
A Review Of Current And Upcoming Treatment Modalities In Non-Alcoholic Fatty Liver Disease And Non-Alcoholic Steatohepatitis
}

This article was published in the following Dove Press journal:

Hepatic Medicine: Evidence and Research

\author{
Surosree Ganguli' \\ Peter DeLeeuw ${ }^{2}$ \\ Sanjaya K Satapathy $\mathbb{D D}^{3}$ \\ 'Division of Internal Medicine, University \\ of Louisville, Louisville, KY 40202, USA; \\ ${ }^{2}$ Division of Internal Medicine, University \\ of Tennessee Health Science Center, \\ Memphis, TN 38163, USA; ${ }^{3}$ Division of \\ Hepatology and Sandra Atlas Bass Center \\ for Liver Diseases, Northwell Health, \\ Manhasset, NY I I030, USA
}

\begin{abstract}
Non-alcoholic fatty liver disease (NAFLD) is the most common cause of chronic liver disease in the West. Non-alcoholic steatohepatitis (NASH) is the progressive form of NAFLD and can lead to cirrhosis, hepatocellular carcinoma, and is associated with increased cardiovascular risks. Multiple components and risk factors are thought to be involved in the pathogenesis of NAFLD and NASH. Optimal therapy has not yet been found, but many advances have been made with the discovery of potential therapeutic options. In this paper, we aim to provide a comprehensive review of approved, studied, and upcoming treatment options for NAFLD and NASH. Non-pharmacologic therapy (lifestyle modifications and bariatric surgery) and pharmacologic therapy are both reviewed. Pharmacologic therapy target components thought to be involved in the pathogenesis of this disease process including insulin resistance, oxidative stress, inflammation, lipid metabolism, and fibrosis are reviewed in this paper. Results of the emerging treatment targets in phase 2 and 3 clinical trials are also included. Keywords: NAFLD, NASH, obesity, cirrhosis, treatment
\end{abstract}

\section{Introduction}

Non-alcoholic fatty liver disease (NAFLD) has become the most common cause of chronic liver disease in the West, affecting up to $30 \%$ of the general population. ${ }^{1,2}$ NAFLD is a spectrum of diseases ranging from simple steatosis to non-alcoholic steatohepatitis (NASH). NASH is a more progressive form of NAFLD associated with increased cardiovascular and liver-related mortality. NASH has increased risk of progression to cirrhosis, end-stage liver disease, and development of hepatocellular carcinoma (HCC).,

Previously, the pathogenesis of NAFLD was proposed to be related to the "twohit" hypothesis with the first hit being hepatic lipid accumulation from risk factors associated with metabolic syndrome leaving the liver susceptible to the second hit, which resulted in activation of inflammation and fibrosis. ${ }^{5}$ Recent findings support a "multiple hit" hypothesis in which a number of parallel processes contribute to the development of progression of NAFLD including gut microbiome dysbiosis, insulin resistance, hormone secretion from adipose tissue, obesity, oxidative stress, and imbalance in inflammatory cytokines. ${ }^{5-7}$ These concurrent "hits" have been translated to potential therapeutic targets now being studied.

Currently, treatment options for NASH are limited. Lifestyle changes with weight loss being the main goal is the foundation of treatment, but it is hard to
Correspondence: Sanjaya K Satapathy Sandra Atlas Bass Center for Liver Diseases \& Transplantation, Division of Hepatology, Department of Medicine, Northshore University Hospital/Northwell Health, 400 Community Drive, Manhasset, NY I I030, USA

$\mathrm{Tel}+15165620510$

$\mathrm{Fax}+15165622688$

Email ssatapat@northwell.edu 
achieve and maintain and is often not enough in morbidly obese patients. There are no current FDA-approved pharmacologic treatment options for NASH emphasizing the need for development of efficacious therapeutic options. However, as the pathogenesis of NASH is further evaluated, targeted treatment options are being studied. Here, we aim to review current and upcoming treatment modalities for the treatment of NAFLD including the progression to NASH.

\section{Non-Pharmacologic Therapy Diet And Weight Loss}

Obesity is an important risk factor in the development of NAFLD and NASH; thus, weight loss is the first-line treatment option for this disease process. ${ }^{8}$ Multiple studies have shown the positive effect of weight loss in the improvement of NAFLD. ${ }^{9-12}$ These studies demonstrated improvement in NAFLD Activity Score (NAS), liver histology, and/or imaging with weight loss. The amount of weight loss required in the treatment of NAFLD has not yet been established, but evidence suggests weight loss of $5 \%$ in NAFLD or $7-10 \%$ in NASH is needed for improvement in histology with even greater weight loss $(>10 \%)$ required in morbidly obese patients. ${ }^{13-15}$ Combination of diet and exercise was found to be most effective in improving NAFLD. ${ }^{14}$ However, the long-term efficacy of diet and lifestyle management in weight loss has been poor given difficulty with compliance. ${ }^{16-18}$

There are limited data on the specific effects of certain diets on NAFLD/NASH. One randomized control trial (RCT) evaluating the effects of the Mediterranean diet compared to low-fat high-carbohydrate diet in nondiabetic biopsy-proven NAFLD patients demonstrated reduction of hepatic steatosis and improvement of insulin sensitivity with Mediterranean diet despite lack of difference in weight loss between diet types. ${ }^{19}$ However, a more recent RCT comparing the Mediterranean diet and low-fat diet found hepatic steatosis and liver enzymes to significantly improve in both groups with no difference in liver fat reduction between groups. As in the previous study, weight loss did not differ between the groups. Unlike the low-fat diet, the Mediterranean diet did improve total cholesterol, serum triglyceride (TG), and glycated hemoglobin (HbAlc), and also had higher adherence rate. ${ }^{20}$ Another randomized study of patients with type 2 diabetes compared the effects of mono-unsaturated fatty acid (MUFA) diet and high-carbohydrate/ high-fiber/low glycemic index (CHO/fiber) diet on liver fat content. ${ }^{21}$ Results from this study demonstrated a significantly lower liver fat content in MUFA diet compared with the $\mathrm{CHO} /$ fiber diet independent of weight loss. There is a lack of consensus in results amongst these studies and they are limited by the small sample sizes as well lack of standardization of study length. Given the historically high rate of long-term non-adherence to lifestyle changes, longer-term studies with a larger sample size are needed.

A review on the effects of different diets on liver fat content and insulin sensitivity demonstrates the multifactorial ways in which macro- and micronutrients contribute to liver fat content. Short-chain fatty acids (SFAs) have been shown to increase liver fat and replacing them with MUFA or N-6 polyunsaturated fatty acids (PUFA) reduces liver fat content. ${ }^{22}$ Of note, the Mediterranean diet is also high in MUFA. ${ }^{22}$ Fiber is another critical macronutrient that can play a role in the pathogenesis of NAFLD. Highfermentable fibers have shown to improve risk factors associated with NAFLD including body weight and insulin resistance. ${ }^{23}$ Low-fermentable fibers have also been shown to decrease risk factors associated with NAFLD including blood glucose and postprandial triglyceride. ${ }^{23}$ In addition, high fiber diets like the Mediterranean diet have also been shown to decrease cardiovascular risk..$^{23,24}$ There is little research on the benefits of specific micronutrients except for Vitamin E, which is discussed later in this review. ${ }^{25}$

An aspect of weight loss that has yet to be widely studied in NAFLD patients is the effects of pharmacologic weight loss agents in the reduction of liver fat content. These drugs can help patients lose $7-10 \%$ of their total body weight, fitting well with the amount of weight loss shown needed to improve liver histology in NASH. ${ }^{26,27}$ One of the criteria to be placed on pharmacologic weight loss agents includes a BMI of greater than 27 , a criterion in which $85 \%$ of the NASH patients qualify. Further, long-term RCTs with assessment of risk factors associated with these agents in patients with NAFLD are required at this time.

\section{Bariatric Surgery}

Bariatric surgery is another option for achieving weight loss in NAFLD and NASH patients. Unlike weight loss through diet and exercise, which can be difficult to achieve and sustain, bariatric surgery provides a longer-term option for weight loss. Bariatric surgery improves NAFLD not only through weight loss but also through the metabolic effects on lipid metabolism and inflammatory pathways associated 
with NAFLD pathophysiology. ${ }^{28,29}$ This procedure is currently indicated in patients with $\mathrm{BMI} \geq 40 \mathrm{~kg} / \mathrm{m}^{2}$ with no comorbidities or a BMI 35 to $39.9 \mathrm{~kg} / \mathrm{m}^{2}$ with at least one serious comorbidity including but not limited to type 2 diabetes, hypertension, obstructive sleep apnea, NAFLD, and NASH. ${ }^{30,31}$

A systematic review of 29 studies in patients undergoing bariatric surgery reported statistically significant improvement in post-operative liver biochemistry (ALT, AST, GGT) and histology. ${ }^{32}$ Another systematic review and meta-analysis by Chavez - Tapia et al reported improvement in liver histology (steatosis, steatohepatitis, and fibrosis) with weight loss after bariatric surgery. ${ }^{33}$ Laursen et al reviewed 13 cohort studies of patients with NAFLD undergoing bariatric surgery with reports of improvement in NASH histology; however, some of the studies also reported worsening histology after bariatric surgery. ${ }^{34}$

As with any invasive procedures, bariatric surgery is not without risks. A systematic review and meta-analysis of the effectiveness and risks of bariatric surgery conducted by Chang et al reported adjustable gastric banding (AGB) with lower mortality and complication rates but higher reoperation rates as well as less significant weight loss as compared to gastric bypass (GB). ${ }^{35}$ Sleeve gastrectomy (SG) was more effective in weight loss than AGB and similar to GB in efficacy. ${ }^{35}$ The mortality rate within 30 days of bariatric surgery was reported to be $0.08 \%$ in the RCTs and $0.31 \%$ after 30 days. ${ }^{35}$ The death rates reported in this review were lower than those reported in previous meta-analysis. ${ }^{35,36}$ Of note, a nationwide inpatient sample (1998-2007) found the mortality of bariatric surgery to be higher in patients with compensated and decompensated cirrhosis. ${ }^{32}$

A recent Cochrane review of the benefits and risks of bariatric surgery for NASH patients was unable to find conclusive data given the lack of RCTs available. ${ }^{33}$ The Longitudinal Assessment of Bariatric Surgery (LABS) consortium reported rare fatal complications following bariatric surgery including sepsis from anastomotic dehiscence, shock secondary to hemorrhage, and cardiopulmonary events. $^{37}$ The leading cause of death after bariatric surgery was found to be due to thromboembolic disease with an incidence of $0.34 \%{ }^{37}$

Long-term complications are dependent on the type of bariatric surgery and have been well outlined in a review by le Roux and Heneghan. ${ }^{38}$ GB can be associated with stricture formation at anastomosis site leading to partial or full obstruction. ${ }^{38,39}$ Internal herniation can complicate both laparoscopic and open bariatric procedures with a rate of $2.5-6.2 \%$ for internal herniation. ${ }^{38,40}$ Patients who have undergone gastric banding are at risk for band-related complications including band slippage and/or erosion as well as esophageal dysmotility, which all result in reintervention rates of up to $48 \% .^{38,41}$ Dietary complications related to bariatric surgery include nutritional deficiencies, dumping syndrome, and postprandial hyperinsulinemic hypoglycemia. ${ }^{38,42-45}$

Large RCTs with longer-term follow-ups are required to better assess the risks and benefits of bariatric surgery in the treatment of NAFLD and NASH.

\section{Pharmacologic Therapy}

\section{Insulin Resistance Targets}

Insulin resistance has been implicated in the pathogenesis of NAFLD with progression to NASH. Therefore, insulin sensitizers are natural targets for treatment intervention. See Table 1 for a comprehensive overview of specific pharmacotherapy therapy target agents studied in NAFLD/NASH.

\section{Metformin}

Metformin, a biguanide, improves insulin resistance by increasing 5'adenosine monophosphate (AMP)-activated protein kinase signaling, in turn, reducing lipid accumulation, glucose output, and TNF- $\alpha$ signaling. ${ }^{46,47}$ Clinical trials of metformin in the treatment of NASH have shown improvement in insulin resistance and liver enzymes. The Treatment of NAFLD in Children (TONIC) trial was an RCT studying the effects of Vitamin E (400 IU twice daily), Metformin (500mg twice daily), or placebo (twice daily) in the treatment of NASH for 96 weeks. ${ }^{48}$ This study demonstrated some improvement in liver histology with metformin treatment, but histologic improvement has been inconsistent between this and other published clinical trials. ${ }^{48}$ Furthermore, a meta-analysis published data from four high-quality RCTs regarding the effect of metformin on NASH and concluded that 6-12 months of metformin along with lifestyle interventions did not improve liver histology aminotransferases in comparison to lifestyle intervention alone. ${ }^{49}$ Although the weight loss-promoting and insulin-sensitizing properties of metformin are desirable, there is lack of evidence in the improvement of liver histology in NAFLD or NASH to suggest this as an adequate treatment option at this time. Ongoing multi-center trials are required for definitive data. 


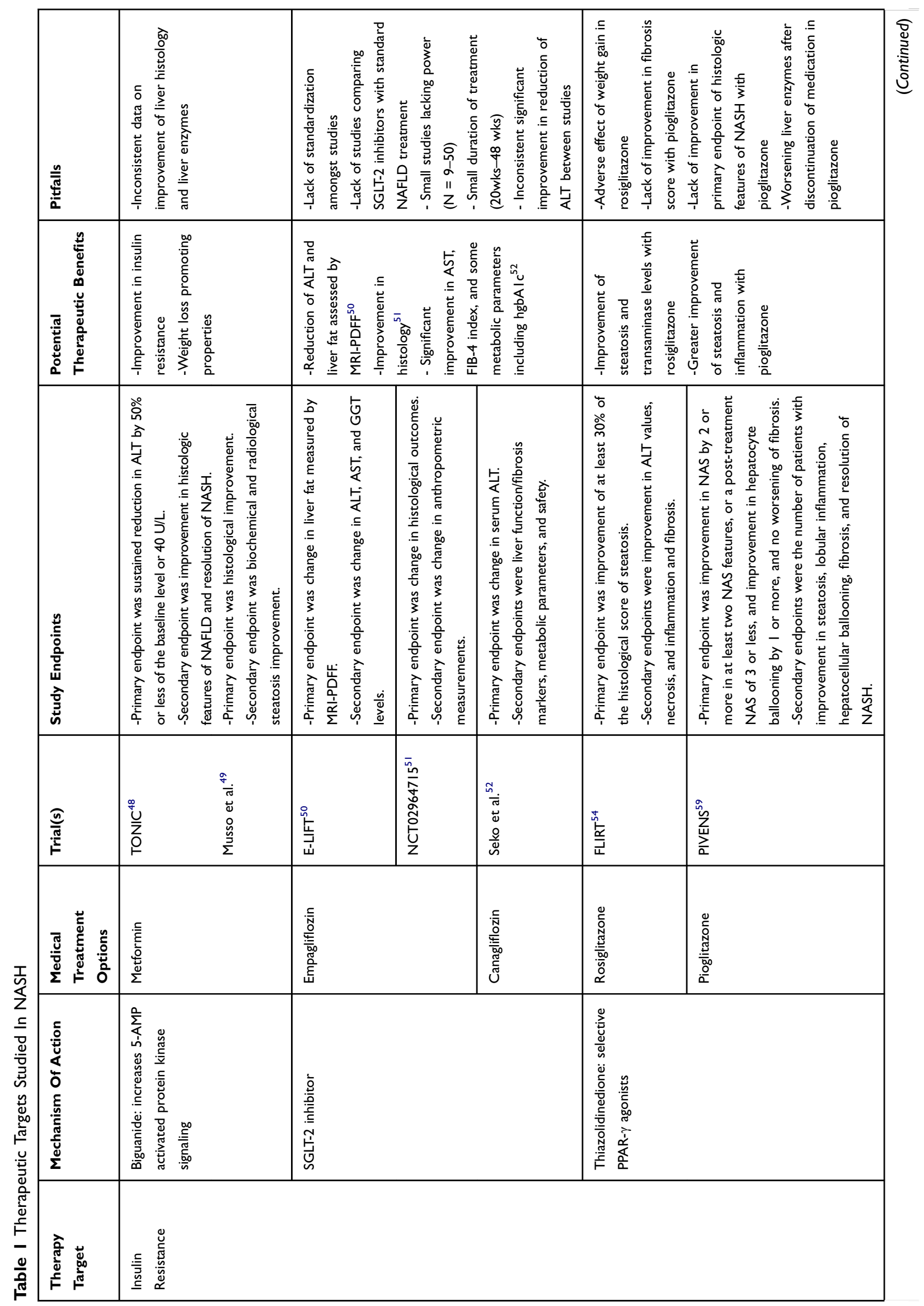




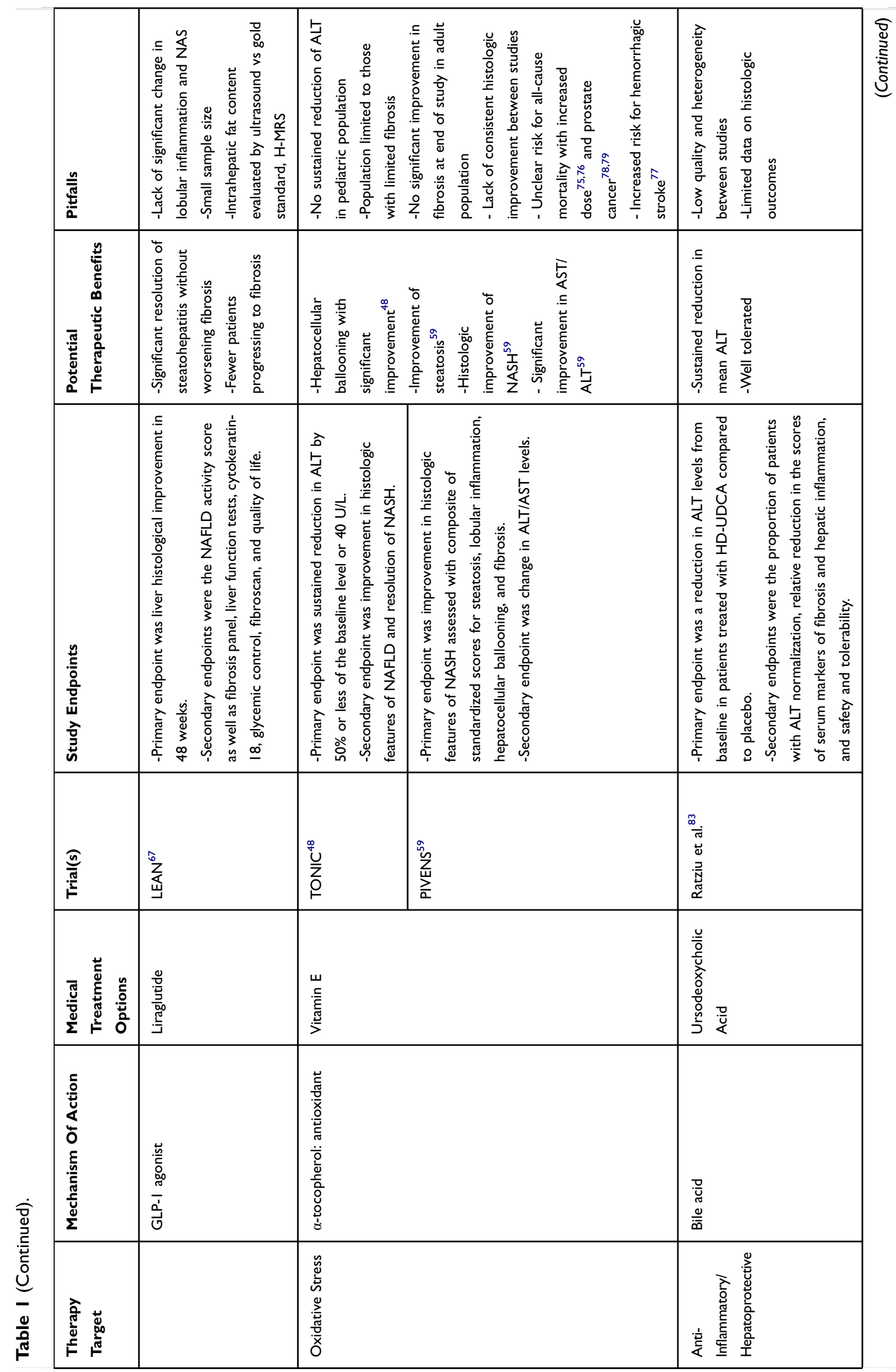




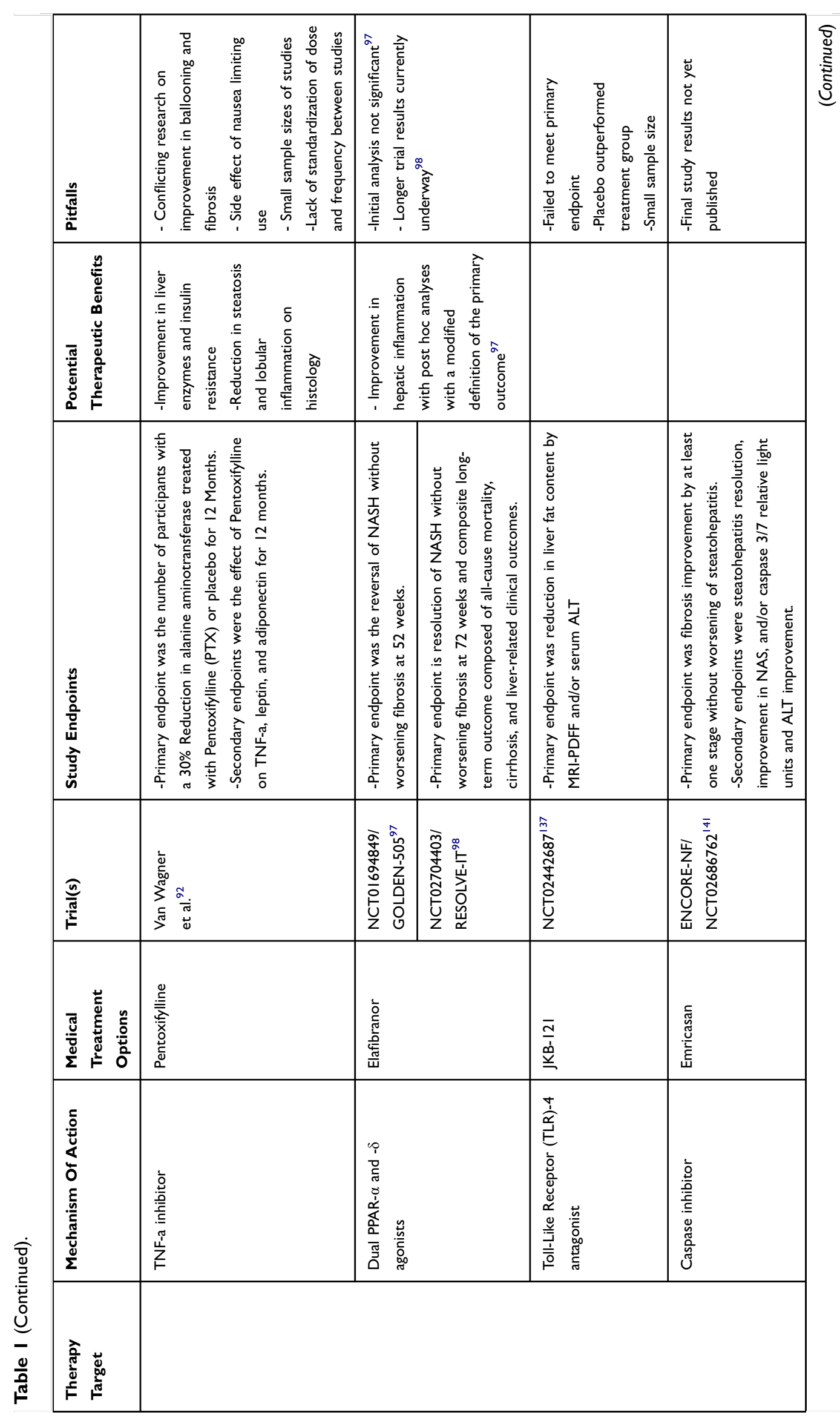




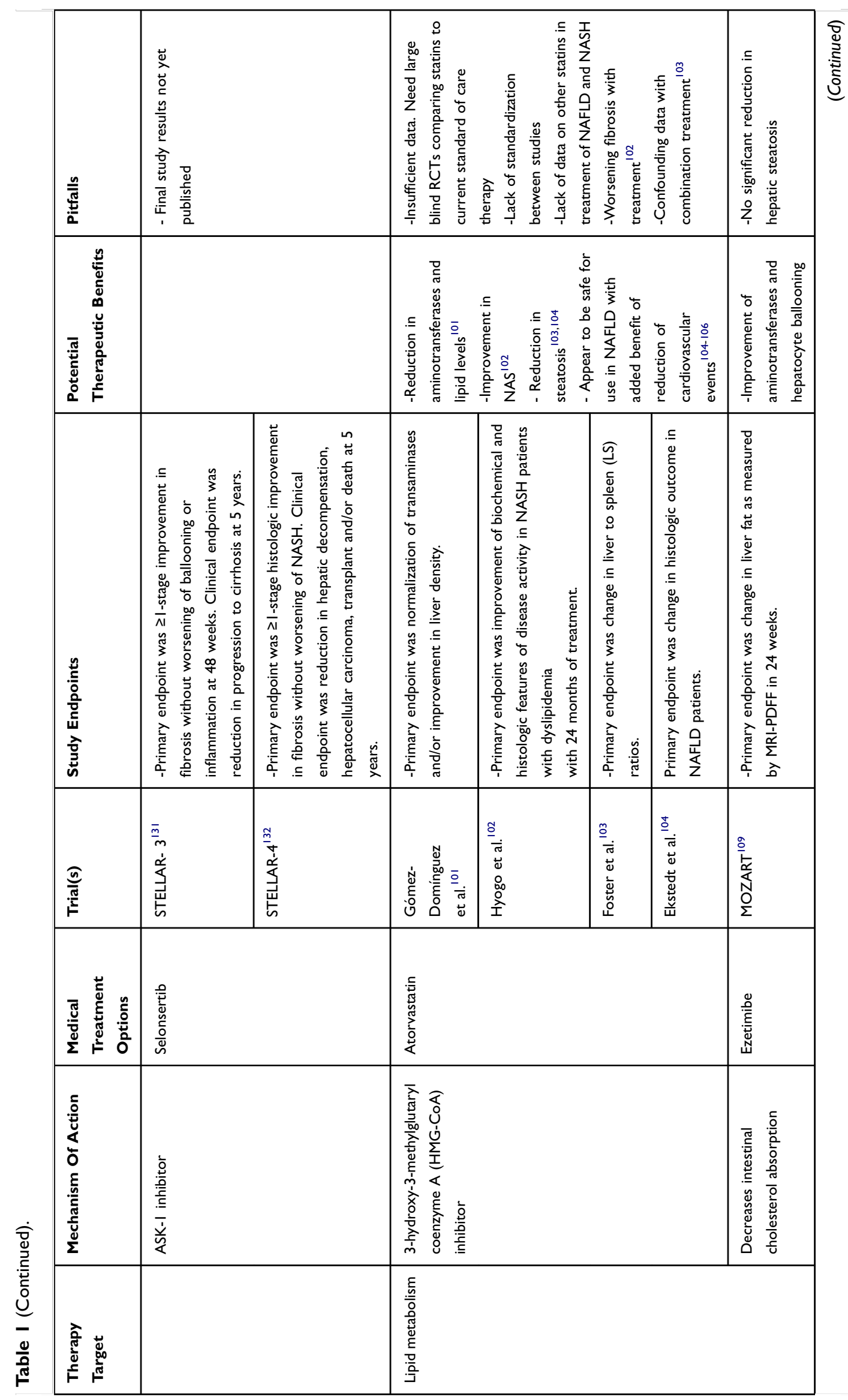




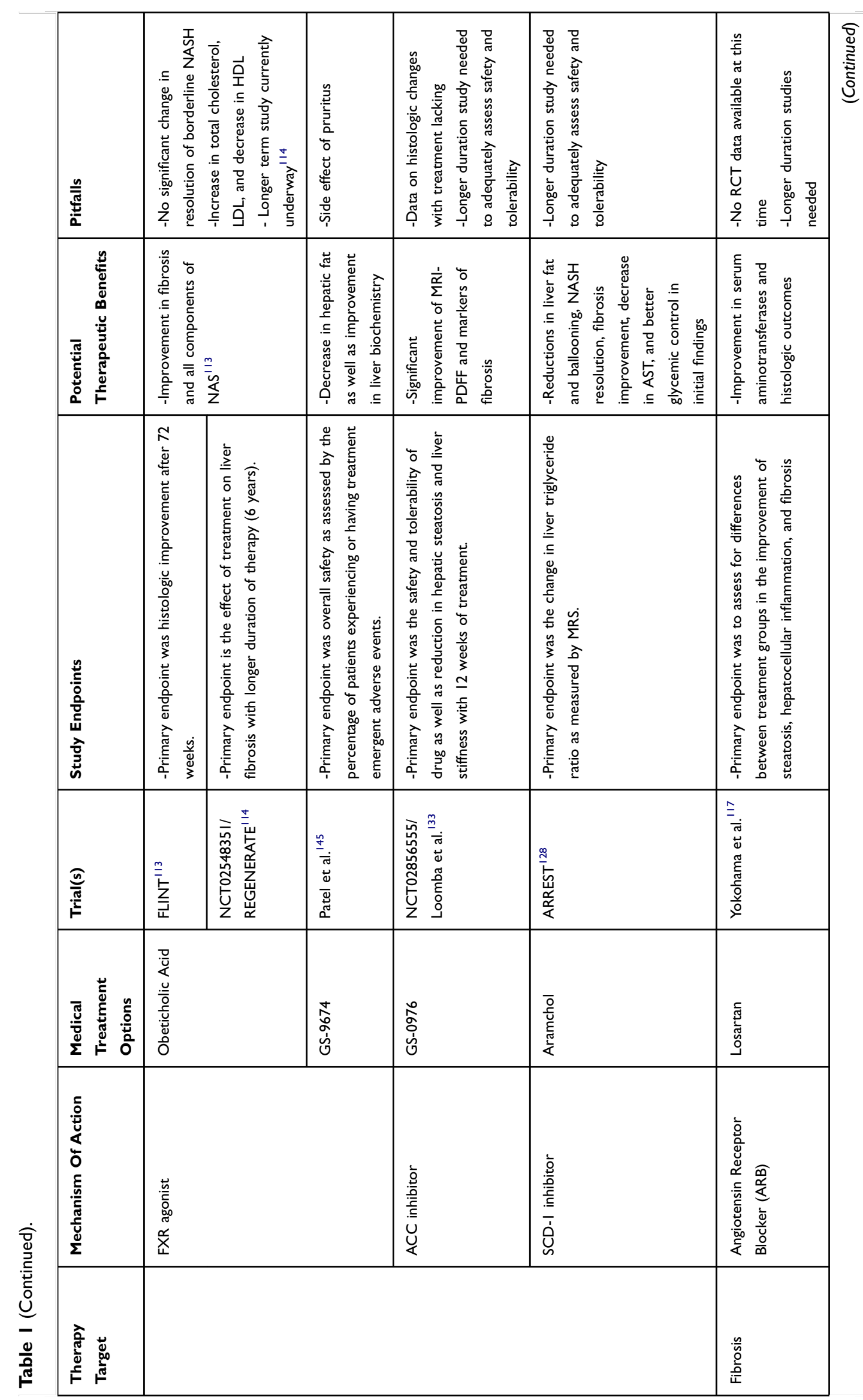




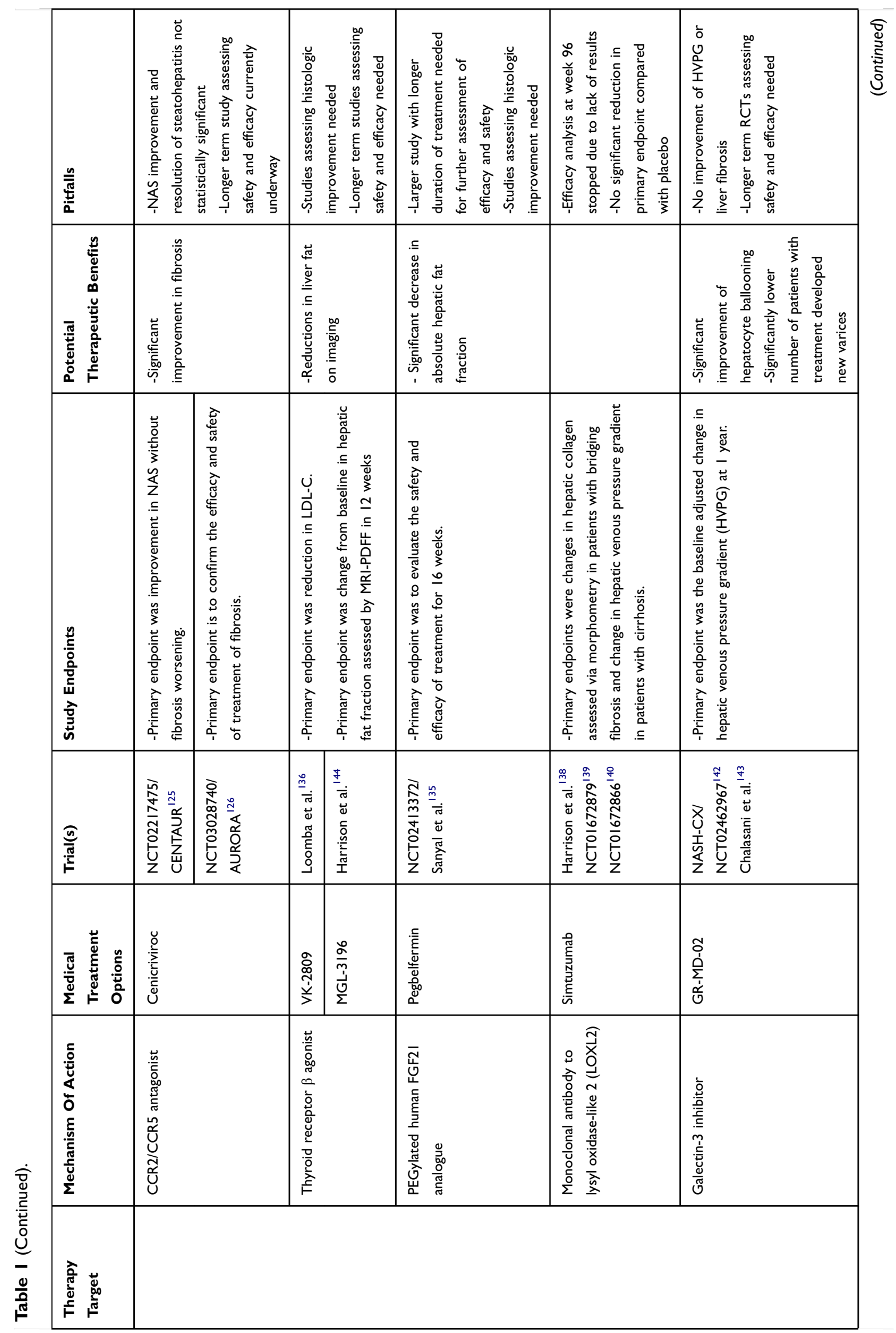




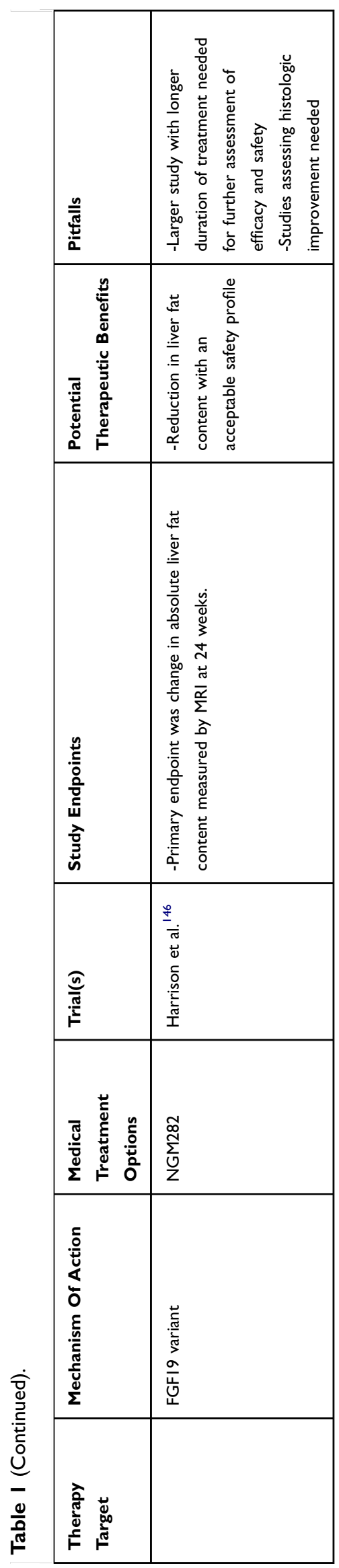

\section{Sodium-Glucose Cotransporter-2 Inhibitors}

Another anti-diabetic agent category that has been studied includes the sodium-glucose cotransporter 2 (SGLT-2) inhibitors. SGLT-2 inhibitors have shown promising results in reducing liver fat content in rodent models, ${ }^{148,149}$ but the data in humans are limited.

The Effect of Empagliflozin on Liver Fat Content in Patients with Type 2 Diabetes (E-LIFT) trial was an RCT evaluating the effects of Empagliflozin versus the standard treatment of Type 2 diabetes mellitus (T2DM) in the treatment of NAFLD. Liver fat, assessed by MRI proton density fat fraction (MRI-PDFF), was significantly reduced compared to control group with a mean difference in fat change between groups $-4 \% .{ }^{50}$ ALT level was also significantly reduced. ${ }^{50}$ Although MRI is non-invasive, there are limitations to its use including the lack of information on inflammation, hepatocyte ballooning, and fibrosis. In addition, patients were using other medications in addition to the treatment medication, which could have led to confounding bias in this study.

Other studies evaluated the effects of Empagliflozin and another SGLT-2 inhibitor, Canagliflozin, in patients with T2DM and NASH and found significant improvement in histology, AST, FIB-4 index, or metabolic parameters. ${ }^{51,52}$ However, the effects of these studies are hard to compare given the lack of standardization between studies including the doses of medications, treatment length, and treatment and control options. Further studies with RCTs comparing SGLT-2 inhibitors with standard NAFLD treatment with longer follow-up periods are needed.

\section{Thiazolidinediones (TZDs)}

TZDs are selective peroxisome proliferator-activated receptor- $\gamma$ (PPAR- $\gamma$ ) agonists that improve insulin resistance and promote fat redistribution from liver and muscle to adipose tissue. ${ }^{53}$

The Fatty Liver Improvement with Rosiglitazone Therapy (FLIRT) trial was the largest trial to evaluate the effects of Rosiglitazone, a TZD, in patients with NASH. Patients had improvement with steatosis and transaminase levels, ${ }^{54}$ but weight gain was a major adverse effect. The FLIRT 2 trial was an extension of the FLIRT trial by an additional 2 years but did not show further improvement in steatosis. ${ }^{55}$

Pioglitazone has shown promise in the treatment of NASH with improvement in steatosis and inflammation compared to placebo in patients with NASH and T2DM. ${ }^{56-59}$ A long term 3-year study of 101 patients 
with NASH and pre-diabetes/T2DM confirmed the longterm safety and efficacy of pioglitazone. ${ }^{58}$ The PIVENS (Pioglitazone, Vitamin E or Placebo for Nonalcoholic Steatohepatitis (PIVENS) trial was a double-blind, RCT evaluating the efficacy of treatment with Vitamin E, Pioglitazone, or placebo for the treatment of NASH in nondiabetic adults. ${ }^{59}$ This trial demonstrated significant improvement of hepatic steatosis and lobular inflammation with pioglitazone use but not with improvement in fibrosis score. ${ }^{59}$ In addition, there was no benefit to using pioglitazone over placebo for the primary outcome of improvement in histologic feature of NASH. Furthermore, liver enzymes worsened after discontinuation of this medication indicating the need for long-term use. Data from studies using pioglitazone have been shown greater benefit than the studies using rosiglitazone. ${ }^{54-56,59}$

There were drawbacks, however, to using this class of drugs including weight gain throughout duration of use as well as risk for heart failure. ${ }^{59,60,61}$ The data support the use of pioglitazone over rosiglitazone in the treatment of T2DM and NASH. ${ }^{54-56,58}$ Pioglitazone improves liver histology in patients with and without T2DM with biopsyproven NASH. AASLD practice guideline recommend its use to treat these patients. ${ }^{147}$ Risks and benefits should be discussed with each patient before starting therapy. However, given the drawbacks and side effects of this class of medications along with lack of improvement in fibrosis, pending further data to support its safety and efficacy, pioglitazone should not be used to treat NAFLD without biopsy-proven NASH. ${ }^{147}$

\section{Glucagon-Like Peptide (GLP)-I Agonists}

GLP-1 agonists are a relatively novel class of antidiabetic medications. They are incretin hormones derived from the gut. Native GLP-1 lowers blood glucose by inducing insulin secretion and reducing glucagon secretion. ${ }^{62,63}$ GLP-1 receptors have been found on hepatocytes leading to further evaluation in their role on the liver. Studies have shown that GLP-1 agonists decrease hepatic steatosis, are hepatoprotective against fatty acid-related death, and reduce fatty acid accumulation. ${ }^{64,65}$ Furthermore, Bernsmeier et al. suggested that GLP-1 secretion is impaired in patients with NAFLD and $\mathrm{NASH}^{66}$

Of the GLP-1 agonists, Liraglutide is the most widely studied medication. The Liraglutide Efficacy and Action in NASH (LEAN) study assessed the effects of treatment with Liraglutide against placebo in patients with biopsy-proven NASH. ${ }^{67}$ The results of this study demonstrated statistically significant resolution of steatohepatitis without worsening fibrosis, which was the primary endpoint. The Liraglutide arm of the study also showed statistically significant fewer patients with progression to fibrosis compared to placebo. However, there was no statistically significant change in lobular inflammation and NAS. The small sample size was a major limitation of this study. Feng et al evaluated treatment with Liraglutide, Metformin, or Gliclazide for 24 weeks in an open-label trial. ${ }^{68}$ All three treatment groups showed a significant decrease in intrahepatic fat with Liraglutide having the greatest reduction. In addition, liver function with AST/ALT levels also improved significantly in the Liraglutide and metformin groups. Small sample size was, again, a major limitation of this study. Further limitations included the use of ultrasonography instead of liver biopsy, the gold standard, to evaluate intrahepatic fat content.

Other GLP-1 agonists being evaluated in the treatment of T2DM and NAFLD/NASH include Exenatide and Semaglutide. Efficacy of Exenatide has not yet been evaluated in the histological outcomes in patients with NAFLD and NASH, but it has shown significant improvement in AST, ALT, and GGT as compared to intensive insulin therapy in a 12 -week study. ${ }^{6}$ Exenatide has also shown to be more effective than metformin in reducing body weight and improving liver enzymes. ${ }^{70}$ Semaglutide is undergoing a placebo-controlled RCT of 372 patients evaluating the efficacy and safety of three different dosage levels of subcutaneous Semaglutide in the treatment of NASH. ${ }^{71}$

The data on GLP-1 is promising but lacking validation at this time. Large-scale placebo-controlled RCTs with assessment of histologic outcomes are needed to evaluate the efficacy and safety of GLP-1 in treatment of NAFLD and NASH. Furthermore, their cost and parenteral route of administration limit the use of these medications in certain populations.

\section{Oxidative Stress Target}

Oxidative stress and impaired antioxidant defense is one of the proposed pathways in the multiple-hits theory in the pathogenesis and progression of NAFLD.

\section{Vitamin E}

Vitamin E ( $\alpha$-tocopherol) is a fat-soluble vitamin that has been extensively studied due to its well-known anti-oxidant properties and role in lipid peroxidation in NASH pathogenesis. ${ }^{72}$ Animal models of NASH demonstrated that Vitamin E decreases levels of TGF- $\beta$, a pro-fibrogenic cytokine, leading to improvement in liver necrosis and 
fibrosis. ${ }^{73,74}$ Vitamin $\mathrm{E}$ therapy was studied in pediatric population in the aforementioned TONIC trial in which hepatocellular ballooning was the only histologic feature of NASH showing significant improvement after treatment (metformin and vitamin E), but neither treatment demonstrated sustained reduction in ALT (the primary outcome) or improvement in steatosis, lobular inflammation, or fibrosis score. ${ }^{48}$ Of note, the patient population included in this study also had only mild fibrosis on histology at the start of treatment. The aforementioned PIVENS trial was similar to the TONIC trial in an adult population demonstrating a higher rate of histologic resolution of NASH at week 96 of treatment with Vitamin E vs pioglitazone (43\% vs $19 \%, \mathrm{p}=0.001) .{ }^{59}$ There was, however, no significant improvement in fibrosis at week 96.

Miller et al conducted a meta-analysis of 11 randomized controlled trials studying the dose-response relationship of Vitamin E treatment with all-cause mortality. ${ }^{75}$ This study suggested an increased all-cause mortality with high-dosage Vitamin E ( $\geq 400 \mathrm{IU} /$ day). Of note, the high dose trials were small and included patients with chronic diseases, which could be contributing to confounding bias. Another larger meta-analysis with 57 trials demonstrated no effect on all-cause mortality with Vitamin $\mathrm{E}$ doses up to 5500 IU/day. ${ }^{76}$ Furthermore, Vitamin E showed increased risk in the incidence of hemorrhagic stroke. ${ }^{77}$ Another concern with the use of Vitamin $\mathrm{E}$ is the increased risk of prostate cancer. However, that risk is unclear given the mixed results between multiple studies. ${ }^{78,79}$

Currently, the European Association for the Study of the Liver (EASL) and the American Association for the Study of Liver Diseases (AASLD) consider Vitamin E a short-term treatment option for non-diabetic adults with biopsy-proven NASH. ${ }^{80,147}$ However, further studies elucidating the long-term safety profile and effectiveness of Vitamin E are required.

\section{Anti-Inflammatory Targets And Hepatoprotectants}

Imbalance in the inflammatory pathways causing hepatocellular damage has been implicated as part of the pathogenesis of NAFLD and NASH.

\section{Ursodeoxycholic Acid (UDCA)}

UDCA is a bile acid that has been evaluated in the treatment of NAFLD given its anti-inflammatory and antiapoptotic properties thought to prevent progression of NAFLD. ${ }^{81}$ A systematic review of 12 RCTs demonstrated promising results for treatment of NASH with UDCA especially in combination with other drugs including vitamin E. ${ }^{82}$ However, the quality of these studies was low and there was significant heterogeneity between results. Ratziu et al performed a 12-month placebo-controlled RCT evaluating the efficacy and safety of high dose UDCA (28-35 mg/kg/day) in biopsy proven NASH with promising results. ${ }^{83}$ They reported significant and sustained reduction in mean ALT and was found to be safe and well tolerated. However, the data on UDCA are limited and further well-designed large RCTs with histologic outcomes are needed to make an informed decision regarding its use in the treatment of NAFLD and NASH.

\section{Pentoxifylline (PTX)}

The imbalance of inflammatory cytokines, such as TNF- $\alpha$, can lead to hepatocellular damage. ${ }^{84}$ PTX inhibits many proinflammatory cytokines include TNF- $\alpha$, reduces oxidized fatty acids, and is thought to have hepatoprotective properties. ${ }^{85-89}$ Small published studies have demonstrated significant improvement of liver enzymes ${ }^{90-92}$ and insulin resistance $^{90}$ as well as improvement in histology ${ }^{92-94}$ with the use of PTX in treatment of NASH. Histologic improvements included significant reduction in steatosis and lobular inflammation. ${ }^{92-94}$ However, there was discordance between improvement in ballooning and fibrosis between these studies. Singh et al completed a meta-analysis evaluating pharmacological interventions in treatment of NASH and found that PTX demonstrated significant improvement in fibrosis compared with placebo whereas Vitamin E, TZDs, and Obeticholic acid (OCA) were not superior to placebo in fibrosis improvement. ${ }^{95}$ PTX was well tolerated other than the side effect of nausea ${ }^{91,94}$ which improved with dose reduction. ${ }^{94}$ The small sample size and lack of standardization regarding the dosage amount and frequency of PTX used between the RCTs and pilot studies posed some limitations. Overall, these initial studies of PTX offer initial promise in its role in the treatment of NASH but cannot be recommended as monotherapy without further evaluation.

\section{Elafibranor}

PPAR- $\alpha$ increases beta-oxidation and decreases steatosis, while PPAR- $\delta$ decreases steatosis and inflammation while increasing insulin sensitivity. Elafibranor is a dual PPAR- $\alpha$ and $-\delta$ agonists. Animal models have demonstrated PPAR to be hepatoprotective by decreasing lipid accumulation, inflammation, and fibrosis. ${ }^{96}$ An RCT evaluated $80 \mathrm{mg}$ or $120 \mathrm{mg}$ of Elafibranor vs placebo in NASH patients for 52 
weeks and found significant improvement in hepatic inflammation in patients with NAS $\geq$ with $120 \mathrm{mg}$ dosing. ${ }^{97}$ However, the primary endpoint of resolution of $\mathrm{NASH}$ without worsening fibrosis was not met. A longer-term phase 3 study of Elafibranor $120 \mathrm{mg}$ daily for 72 weeks vs placebo is currently underway to evaluate its efficacy in improving histologic outcomes. ${ }^{98}$

\section{Lipid Metabolism Targets}

NAFLD and NASH are hepatic manifestations of metabolic syndrome. Dyslipidemia and abnormal lipid metabolism are thought to be a part of the pathogenesis of development of NASH.

\section{Lipid-Lowering Agents}

Statins are used to treat dyslipidemia and are thought to also have anti-oxidant and anti-inflammatory effects. ${ }^{99,100}$ A pilot study with atorvastatin treatment demonstrated significant reduction in serum aminotransferase and lipid levels in patients with NAFLD. ${ }^{101}$ However, this study did not evaluate histologic outcomes. An open-label study evaluating atorvastatin in patients with biopsy-proven NASH reported significant improvement in NAS, but $24 \%$ of the patients had worsening fibrosis. ${ }^{102}$ An RCT evaluating atorvastatin in combination with antioxidants such as Vitamin $\mathrm{C}$ and $\mathrm{E}$ effectively reduced the risk of steatosis by $71 \%$ after 4 years of treatment in patients with NAFLD. ${ }^{103}$ Many patients with NAFLD and NASH usually receive statins regardless given their cardiovascular risk factors. Although there have been concerns regarding the safety of statin use in patients with liver disease given the risk of hepatotoxicity, recent studies demonstrated statins to be relatively safe in patients with NAFLD. ${ }^{104-106}$

Ezetimibe is another medication used in the treatment of hypercholesterolemia. It has been found to prevent hepatic steatosis and decrease hepatic insulin resistance in mice model of hepatic steatosis. ${ }^{107}$ Nakade et al completed a meta-analysis including 6 studies and reported improvement of serum aminotransferases and hepatocyte ballooning in patients with NAFLD receiving ezetimibe treatment. ${ }^{108}$ The Magnetic Resonance Imaging and Elastography in Ezetimibe Versus Placebo for the Assessment of Response to Treatment in NASH (MOZART) trial, however, did not show significant reduction in hepatic steatosis with ezetimibe treatment. ${ }^{109}$ However, liver biopsy was not performed and steatosis was measured through MRI-derived proton density fat fraction (MRI-PDFF).
These lipid-lowering agents are not recommended as monotherapy for NAFLD and NASH at this time without further evaluation with large RCT evaluating histologic outcomes.

\section{Farnesoid X Receptor (FXR) Agonists}

FXR has been identified as a receptor for bile acids and has been found to regulate lipid metabolism and modulate glucoregulatory pathways. ${ }^{110,111}$ This is a relatively novel class of drug target being studied in the treatment of NAFLD and NASH. Obeticholic acid (OCA) is a FXR agonist and bile acid analogue with a 100-fold greater potency on FXR. ${ }^{112}$ The Farnesoid X Receptor Ligand Obeticholic Acid in Nash treatment (FLINT) trial was a multicenter RCT that evaluated the histologic improvement with OCA $25 \mathrm{mg}$ daily treatment in non-cirrhotic patients with NASH for 72 weeks. ${ }^{113}$ Results from this trial demonstrated fibrosis improvement without worsening of steatohepatitis in OCA (43\% vs $21 \%$ in placebo, $\mathrm{p}<0.001$ ) as well as improvement in all components of NAS as well as fibrosis. However, OCA group had an increase in total cholesterol and LDL along with a drop in HDL compared with placebo. Further studies evaluating the long-term safety of this medication are required. One such study, the Randomized Global Phase 3 Study to Evaluate the Impact on NASH With Fibrosis of Obeticholic Acid Treatment (REGENERATE) trial, is a current long-term 6-year duration study evaluating the effect of treatment with OCA $10 \mathrm{mg}$ vs $25 \mathrm{mg}$ vs placebo on liver fibrosis. ${ }^{114}$ Overall, OCA seems to be another promising agent in the treatment of NASH.

\section{Fibrosis Targets}

Targeting the progression of fibrosis is another pharmacologic option in preventing the progression of NAFLD and $\mathrm{NASH}$.

\section{Angiotensin Receptor Blockers (ARBs)}

ARBs are a class of medications frequently used in the treatment of hypertension especially in the setting of diabetes. Angiotensin II is thought to promote liver fibrosis by activating transforming growth factor- $\beta$ (TGF- $\beta$ ) and Tolllike receptor-4 (TLR-4) signaling. ${ }^{115,116}$ Uncontrolled trials with Losartan, an ARB, demonstrated significant improvement in serum aminotransferases as well as in histologic outcomes. ${ }^{117,118}$ However, an open-label trial evaluating concurrent treatment with Rosiglitazone and Losartan did not show an improvement in histopathology as compared to 
treatment with rosiglitazone alone. ${ }^{119}$ Another ARB, Telmisartan, was found to significantly reduce serum ALT levels and improve insulin sensitivity as well as steatosis, necroinflammation, and fibrosis with greater efficacy than valsartan. $^{120}$ A study involving rats with T2DM demonstrated reduction in hepatic fibrosis and steatosis as well as decreased tissue expression of TNF- $\alpha$ with administration of the ARB, Valsartan $(15 \mathrm{mg} / \mathrm{kg} /$ day $)$, for four months. ${ }^{121}$ Further placebo-controlled, large-scale RCTs evaluating of histologic outcomes are required at this time to confirm the therapeutic effects of ARBs in treatment of NAFLD and NASH.

\section{Chemokine Receptor (CCR) Antagonists}

CCRs are expressed on immune cells and stimulate hepatic stellate cells promoting fibrosis. Cenicriviroc (CVC) is a CCR2 and CCR5 inhibitor. Animal models of NASH demonstrated decreased fibrosis and inflammation with CVC treatment. ${ }^{122-124}$ The CENTAUR trial was a phase $2 \mathrm{~b}$ randomized double-blind multinational study in patients with NASH undergoing treatment with CVC. ${ }^{125}$ Significant improvement in fibrosis was seen in twice as many patients in the treatment arm compared with placebo (20\% vs $10 \%$, $\mathrm{p}=0.02) .{ }^{125}$ There was also NAS improvement as well as resolution of steatohepatitis, but these were not statistically significant. Currently, phase 3 (NCT03028740) of this study is underway to confirm the efficacy and safety of CVC in treatment of fibrosis in NASH. ${ }^{126}$

\section{Ongoing Trials}

Many new pharmacologic targets are being evaluated currently for the treatment of NAFLD and NASH. Here, we will summarize current ongoing trials of potential therapeutic targets in the treatment of NASH. See Table 1 for comprehensive overview NAFLD/NASH pharmacotherapy target agents including agents currently being studied which have not been discussed in the following text given lack of data at this time.

\section{Aramchol}

Stearoyl-CoA desaturase 1 (SCD1) is a key enzyme involved in triglyceride biosynthesis. Aramchol inhibits SCD1 and enhances fatty acid oxidation and has been shown to decrease steatohepatitis and fibrosis in mice models of NASH. ${ }^{127}$ Recent Phase 2 one-year results from the RCT ARREST trial evaluated the role of aramchol $600 \mathrm{mg}, 400 \mathrm{mg}$, or placebo in biopsy-proven NASH and found the $600 \mathrm{mg}$ arm of the study with significantly more patients with dose-dependent reduction in liver fat and ballooning, NASH resolution as well as fibrosis improvement. ${ }^{128}$ There was also decrease in serum aminotransferases and better glycemic control. Further testing in phase 3 trial is underway. ${ }^{129}$

\section{Selonsertib}

Inhibition of apoptosis signaling-regulating kinase 1 (ASK 1) has been shown to improve inflammation and fibrosis in animal models of NASH. In a 24-week open-label phase 2 RCT, the safety and efficacy of $6 \mathrm{mg}$ or $8 \mathrm{mg}$ Selonsertib, a selective ASK 1 inhibitor, alone or in combination with Simtuzumab (SIM) or SIM alone in patients with NASH and stage 2 or 3 fibrosis was evaluated. ${ }^{130}$ Progression to cirrhosis was lowest in the $18 \mathrm{mg}$ treatment group at $3 \%$ vs $7 \%$ in the $6 \mathrm{mg}$ group vs $20 \%$ in the SIM group. Phase 3 trials of Selonsertib in patients with NASH and F3 fibrosis (STELLAR-3) and compensated cirrhosis (STELLAR-4) have been completed, but final results have yet to be published. ${ }^{131,132}$ In these studies, the efficacy of daily Selonsertib $18 \mathrm{mg}$, $6 \mathrm{mg}$, or placebo was evaluated with the primary endpoint being $\geq 1$ point decrease in fibrosis stage without worsening of ballooning or inflammation at 48 weeks. The clinical endpoint at year 5 was to be the reduction in progression to cirrhosis in STELLAR-3 and hepatic decompensation, hepatocellular carcinoma, transplant, and/or death in STELLAR-4. However, the study was halted prior to the set clinical endpoint due to inefficacy, as both the the studies did not meet the pre-specified week 48 primary endpoint of $\mathrm{a} \geq 1$ stage histologic improvement in fibrosis without worsening of NASH. The final results have not been published at this time.

\section{Acetyl-Coenzyme Carboxylase (ACC) Inhibitor (GS-0976)}

ACC is the rate-limiting step in de novo lipogenesis. The safety and efficacy of GS-0976, an inhibitor of ACC, were evaluated in a phase 2 placebo-controlled RCT. Patients received $20 \mathrm{mg}, 5 \mathrm{mg}$, or placebo daily for 12 weeks. The $20 \mathrm{mg}$ group was found to have significant improvement in MRI-PDFF and markers of fibrosis. ${ }^{133}$

\section{Pegbelfermin (BMS-986036)}

Pegbelfermin is a PEGylated human fibroblast growth factor 21 (FGF21) analogue that has been shown to improve markers of metabolism and liver fibrosis in mouse model of NASH. ${ }^{134}$ A phase 2 placebo-controlled RCT evaluated the safety and efficacy of subcutaneous injections of placebo daily, $10 \mathrm{mg}$ pegbelfermin daily, or 20mg pegbelfermin once weekly for 16 weeks of total therapy in patients with NASH. ${ }^{135}$ Results demonstrated significant decrease in absolute hepatic fat fraction in $10 \mathrm{mg}$ treatment group $(-6.8 \%$ vs $-1.3 \%, \mathrm{p}=0.0004)$ 
and in $20 \mathrm{mg}$ treatment group $(-5.2 \%$ vs $-1.3 \%,=0.008)$ compared with placebo. Further assessment of efficacy in improvement of histologic endpoints in a larger study is warranted.

\section{VK2809}

Thyroid hormones, especially the $\beta$ isoform, regulate lipid metabolism especially via specific hepatic receptor. VK2809 is a novel liver-directed thyroid receptor $\beta$ receptor agonist whose safety and efficacy were evaluated in a phase 2 RCT where patients with NAFLD were administered oral $5 \mathrm{mg}$ daily, $10 \mathrm{mg}$ daily, $10 \mathrm{mg}$ every other day (QOD), or placebo over a 12-week period. ${ }^{136}$ Results demonstrated significant reductions in liver fat content assessed by MRI-PDFF as compared with placebo $(53.8 \%$ in $5 \mathrm{mg}$ QD vs $56.5 \%$ in $10 \mathrm{mg}$ QOD vs $-59.7 \% 10 \mathrm{mg}$ QD vs $9.4 \%$ in placebo, $\mathrm{p}=0.0001$ vs 0.0018 vs 0.0004 vs 0.0003 ). As high as $100 \%$ of the patients also demonstrated $\geq 30 \%$ reduction in liver fat at 12 weeks with $5 \mathrm{mg}$ QD dosing vs $76.9 \%$ with $10 \mathrm{mg}$ QOD dosing vs $90.9 \%$ in $10 \mathrm{mg}$ QD dosing vs $16.7 \%$ in placebo $(\mathrm{p}=$ 0.0002 vs 0.0048 vs 0.0006 ). These robust improvements are promising and prompt further evaluation.

\section{Conclusions}

There are currently no FDA approved drugs for the treatment of NASH. The current mainstay of therapy is diet and lifestyle modification. Given the slow rate of progression of hepatic fibrosis in most patients with NASH it is emphasized that treatment be aimed at controlling associated metabolic risk factors rather than pharmacological intervention. Since, weight loss is associated with improvements in serum aminotransferase activities as well as components of metabolic syndrome, this is commonly recommended. Weight loss through bariatric surgery has shown promising results. Patients at risk for fibrosis progression and those who already have advanced disease should be given opportunity to particiapte in clinical trials. In patients included in clinical trials, therapy should not only be aimed at improving the aminotransferses and NAS activity score, but also to halt or reverse fibrosis progression. From a practical stand point, while dealing with such patients in the clinical practice, we would suggest intervention based on the patients associated metabolic risk profile. The gut-liver axis, another pathway in the pathogenesis of NASH has been explored, and this apparoch may lead to many newer therapies in the future targeting gut dysbiosis.

TZDs and Vitamin E seem to have the most abundant promising data but has not shown any benefit in fibrosis improvement which is the key marker of long term outcome in NASH patients. Many of the other drugs reviewed in this paper, including the newer drug targets, have shown very promising results with regards to their safety and efficacy in treating NAFLD/NASH, but an unequivocal proven benefit is yet to be shown. Before progress in treatment can be achieved, additional rigorous research aimed at elucidating the key pathways of its pathogenesis is warranted. Future clinical and preclinical studies on existing and newer agents hopefully will pave the way for treatment of this increasingly prevalent disease.

\section{Author Contributions}

Sanjaya K Satapathy, Surosree Ganguli, and Peter DeLeeuw made substantial contributions to conception and design, acquisition of data, and analysis and interpretation of data. All assisted in drafting the article or revising it critically for important intellectual content. They have all given final approval of the version to be published and have agreed to be accountable for all aspects of the work in ensuring that questions related to the accuracy or integrity of any part of the work are appropriately investigated and resolved.

\section{Disclosure}

Dr Sanjaya K Satapathy reports grants from Gilead, Intercept, Shire, Allergan, Bayer, Conatus, Exact Sciences, Dova, Biotest, and Genfit, served on the Speakers Bureau for Alexion, Intercept, and Dova, and served on the Advisory board for Gilead, Bayer, and Dova, outside the submitted work. The authors report no other conflicts of interest in this work.

\section{References}

1. Browning JD, Szczepaniak LS, Dobbins R, et al. Prevalence of hepatic steatosis in an urban population in the United States: impact of ethnicity. Hepatology. 2004;40(6):1387-1395. doi:10.1002/hep.20466

2. Loomba R, Sanyal AJ. The global NAFLD epidemic. Nat Rev Gastroenterol Hepatol. 2013;10(11):686-690. doi:10.1038/nrgastro. 2013.171

3. Fan JG. Impact of non-alcoholic fatty liver disease on accelerated metabolic complications. J Dig Dis. 2008;9:63-67. doi:10.1111/ j.1751-2980.2008.00323.x

4. Angulo P. Long-term mortality in nonalcoholic fatty liver disease: is liver histology of any prognostic significance? Hepatology. 2010;51:373-375. doi:10.1002/hep.23521

5. Buzzetti E, Pinzani M, Tsochatzis EA. The multiple-hit pathogenesis of non-alcoholic fatty liver disease (NAFLD). Metabolism. 2016;65 (8):1038-1048. doi:10.1016/j.metabol.2015.12.012

6. Musso G, Cassader M, Gambino R. Non-alcoholic steatohepatitis: emerging molecular targets and therapeutic strategies. Nat Rev Drug Discov. 2016;15(4):249-274. doi:10.1038/nrd.2015.3 
7. Kirpich IA, Marsano LS, McClain CJ. Gut-liver axis, nutrition, and non-alcoholic fatty liver disease. Clin Biochem. 2015;48:923-930. doi:10.1016/j.clinbiochem.2015.06.023

8. Neuschwander-Tetri BA. Lifestyle modification as the primary treatment of NASH. Clin Liver Dis. 2009;13:649-665. doi:10.1016/j. cld.2009.07.006

9. Huang MA, Greenson JK, Chao C, et al. One-year intense nutritional counseling results in histological improvement in patients with nonalcoholic steatohepatitis: a pilot study. Am J Gastroenterol. 2005;100 (5):1072-1081. doi:10.1111/j.1572-0241.2005.41334.x

10. Promrat K, Kleiner DE, Niemeier HM, et al. Randomized controlled trial testing the effects of weight loss on nonalcoholic steatohepatitis. Hepatology. 2010;51(1):121-129. doi:10.1002/hep.23276

11. Patel NS, Doycheva I, Peterson MR, et al. Effect of weight loss on magnetic reso- nance imaging estimation of liver fat and volume in patients with nonalcoholic steatohepatitis. Clin Gastroenterol Hepatol. 2015;13(3):561-8.e1. doi:10.1016/j.cgh.2014.08.039

12. Vilar-Gomez E, Martinez-Perez Y, Calzadilla-Bertot L, et al. Weight loss via life- style modification significantly reduces features of nonalcoholic steatohepatitis. Gastroenterology. 2015;149 (2):367-78.e5. doi:10.1053/j.gastro.2015.04.005

13. Hannah WN Jr, Harrison SA. Effect of Weight Loss, Diet, Exercise, and Bariatric Surgery on Nonalcoholic Fatty Liver Disease. Clin Liver Dis. 2016;20(2):339-350. doi:10.1016/j.cld.2015.10.008

14. Kenneally S, Sier JH, Moore JB. Efficacy of dietary and physical activity intervention in non-alcoholic fatty liver disease: a systematic review. BMJ Open Gastroenterol. 2017;4:e000139.Published 2017. doi:10.1136/bmjgast-2017-000139

15. Hohenester $\mathrm{S}$, Christiansen $\mathrm{S}$, Nagel J, et al. Lifestyle intervention for morbid obesity: effects on liver steatosis, inflammation, and fibrosis. $\mathrm{Am}$ J Physiol Gastrointest Liver Physiol. 2018. doi:10.1152/ ajpgi.00044.2018

16. Foster GD, Wyatt HR, Hill JO, et al. A randomized trial of a lowcarbohydrate diet for obesity. N Engl J Med. 2003;348:2082-2090. doi:10.1056/NEJMoa022207

17. Sacks FM, Bray GA, Carey VJ, et al. Comparison of weight-loss diets with different compositions of fat, protein, and carbohydrates. N Engl J Med. 2009;360:859-873. doi:10.1056/NEJMoa0804748

18. Erlichman J, Kerbey AL, James WP. Physical activity and its impact on health outcomes. Paper 2. prevention of unhealthy weight gain and obesity by physical activity: an analysis of the evidence. Obes Rev. 2002;3:273-287.

19. Ryan MC, Itsiopoulos C, Thodis $\mathrm{T}$, et al. The Mediterranean diet improves hepatic steatosis and insulin sensitivity in individuals with non-alcoholic fatty liver disease. J Hepatol. 2013;59(1):138143. doi:10.1016/j.jhep.2013.02.012

20. Properzi C, O'Sullivan TA, Sherriff JL, et al. Ad libitum mediterranean and low-fat diets both significantly reduce hepatic steatosis: a randomized controlled trial. Hepatology. 2018;68(5):1741-1754. doi:10.1002/hep.30076

21. Bozzetto L, Prinster A, Annuzzi G, et al. Liver fat is reduced by an isoenergetic MUFA diet in a controlled randomized study in type 2 diabetic patients. Diabetes Care. 2012;35(7):1429-1435. doi:10.2337/dc12-0033

22. Yki-Järvinen H. Nutritional modulation of non-alcoholic fatty liver disease and insulin resistance. Nutrients. 2015;7(11):9127-9138. doi:10.3390/nu7115454

23. Bozzetto L, Costabile G, Della PG, et al. Dietary fibre as a unifying remedy for the whole spectrum of obesity-associated cardiovascular risk. Nutrients. 2018;10(7). doi:10.3390/nu10070943

24. Estruch R, Ros E, Salas-Salvadó J, et al. Primary prevention of cardiovascular disease with a mediterranean diet. $N$ Engl J Med. 2013;368(14):1279-1290. doi:10.1056/NEJMoa1200303
25. Della Pepa G, Vetrani C, Lombardi G, Bozzetto L, Annuzzi G, Rivellese AA. Isocaloric dietary changes and non-alcoholic fatty liver disease in high cardiometabolic risk individuals. Nutrients. 2017;9(10). doi:10.3390/nu9101065

26. Do A, Kuszewski EJ, Mehal WZ. Incorporating weight loss medications into hepatology practice for nonalcoholic steatohepatitis. Hepatology. 2019. doi:10.1002/hep.30658

27. Khera R, Murad MH, Chandar AK, et al. Association of pharmacological treatments for obesity with weight loss and adverse events: a systematic review and meta-analysis. Jama. 2016;315 (22):2424-2434. doi:10.1001/jama.2016.7602

28. Klein S, Mittendorfer B, Eagon JC, et al. Gastric bypass surgery improves metabolic and hepatic abnormalities associated with nonalcoholic fatty liver disease. Gastroenterology. 2006;130:1564-1572. PMID: 16697719. doi:10.1053/j.gastro. 2006.01.042

29. Viana EC, Araujo-Dasilio KL, Miguel GP, et al. Gastric bypass and sleeve gastrectomy: the same impact on IL-6 and TNF- $\alpha$. Prospective clinical trial. Obes Surg. 2013;23:1252-1261. PMID: 23475776. doi:10.1007/s11695-013-0894-2

30. NIH conference. Gastrointestinal surgery for severe obesity. Consensus development conference panel. Ann Intern Med. 1991;115:956-961.

31. Burguera B, Agusti A, Arner P, et al. Critical assessment of the current guidelines for the management and treatment of morbidly obese patients. J Endocrinol Invest. 2007;30:844-852. doi:10.1007/ BF03349226

32. Bower G, Toma T, Harling L, et al. Bariatric surgery and nonalcoholic fatty liver disease: a systematic review of liver biochemistry and histology. Obes Surg. 2015. doi:10.1007/s11695-0151691-x

33. Chavez-Tapia NC, Tellez-Avila FI, Barrientos-Gutierrez T, MendezSanchez N, Lizardi-Cervera J, Uribe M. Bariatric surgery for nonalcoholic steatohepatitis in obese patients. Cochrane Database Syst Rev. 2010;1:CD007340. doi:10.1002/14651858.CD007340.pub2

34. Laursen TL, Hagemann CA, Wei C, et al. Bariatric surgery in patients with non-alcoholic fatty liver disease - from pathophysiology to clinical effects. World J Hepatol. 2019;11(2):138-149. doi:10.4254/wjh.v11. i2.138

35. Chang S, Stoll CRT, Song J, et al. The effectiveness and risks of bariatric surgery: an updated systematic review and meta-analysis, 2003-2012. JAMA Surg. 2014;149(3):275-287. doi:10.1001/jamasurg/2013.3654

36. Buchwald H, Avidor Y, Braunwald E, et al. Bariatric surgery: a systematic review and meta-analysis. JAMA. 2004;292(14):17241737. doi:10.1001/jama.292.14.1724

37. Flum DR, Belle SH, King WC, et al. Perioperative safety in the longitudinal assessment of bariatric surgery. $N$ Engl J Med. 2009;361:445-454. doi:10.1056/NEJMoa0901836

38. Le Roux CW, Heneghan HM. Bariatric surgery for obesity. Med Clin North Am. 2018;102(1):165-182. doi:10.1016/j.mcna.2017.08.011

39. Carrodeguas L, Szomstein S, Zundel N, et al. Gastrojejunal anastomotic strictures following laparoscopic Roux-en-Y gastric bypass surgery: analysis of 1291 patients. Surg Obes Relat Dis. 2006;2:9297. doi:10.1016/j.soard.2005.10.014

40. Bauman RW, Pirrello JR. Internal Hernia at Peterson's space after laparoscopic Roux-en-Y gastric bypass: $6.2 \%$ incidence without closure- a single surgeon series of 1047 cases. Surg Obes Relat Dis. 2009;5:565-570. doi:10.1016/j.soard.2008.10.013

41. Arapis K, Tammaro P, Parenti LR, et al. Long-term results after laparoscopic adjustable gastric banding for morbid obesity: 18-year follow-up in a single university unit. Obes Surg. 2016;26:22552256. doi: $10.1007 / \mathrm{s} 11695-016-2267-0$ 
42. Toh SY, Zarshenas N, Jorgensen J. Prevalence of nutrient deficiencies in bariatric patients. Nutrition. 2009;25:1150-1156. doi:10.1016/j. nut.2009.03.012

43. Parrot J, Frank L, Rabena R, et al. American Society for Metabolic and Bariatric Surgery integrated health nutritional guidelines for the surgical weight loss patient 2016 update: micronutrients. Surg Obes Relat Dis. 2017;13:727-741. doi:10.1016/j.soard.2016.12.018

44. Hammer HF. Medical complications of bariatric surgery: focus on malabsorption and dumping syndrome. Dig Dis. 2012;30:182-186. doi:10.1159/000336681

45. Marsk R, Jonas E, Rasmussen F, et al. Nationwide cohort study of post-gastric bypass hypoglycemia including 5,040 patients undergoing surgery for obesity in 1986-2006 in Sweden. Diabetologia. 2010;53:2307-2311. doi:10.1007/s00125-010-1798-5

46. Zhou G, Myers R, Li Y, et al. Role of AMP-activated protein kinase in mechanism of metformin action. J Clin Invest. 2001;108:11671174. doi:10.1172/JCI13505

47. Lin HZ, Yang SQ, Chuckaree C, Kuhajda F, Ronnet G, Diehl AM. Metformin reverses fatty liver disease in obese, leptin-deficient mice. Nat Med. 2000;6:998-1003. doi:10.1038/79697

48. Lavine JE, Schwimmer JB, Van Natta ML, et al. Effect of vitamin $\mathrm{E}$ or metformin for treatment of nonalcoholic fatty liver disease in children and adolescents: the TONIC randomized controlled trial. JAMA. 2011;305(16):1659-1668. doi:10.1001/jama.2011.520

49. Musso G, Gambino R, Cassader M, Pagano G. A meta-analysis of randomized trials for the treatment of nonalcoholic fatty liver disease. Hepatology. 2010;52(1):79-104. doi:10.1002/hep.23623

50. Kuchay MS, Krishan S, Mishra SK, et al. Effect of empagliflozin on liver fat in patients with type 2 diabetes and nonalcoholic fatty liver disease: a randomized controlled trial (E-LIFT Trial). Diabetes Care. 2018;41(8):1801-1808. doi:10.2337/dc18-0165

51. Lai LL, Vethakkan SR, Nik Mustapha NR, Mahadeva S, Chan WK. Empagliflozin for the treatment of nonalcoholic steatohepatitis in patients with type 2 diabetes mellitus. Dig Dis Sci. 2019. doi:10.1007/s10620-019-5477-1

52. Seko Y, Nishikawa T, Umemura A, et al. Efficacy and safety of canagliflozin in type 2 diabetes mellitus patients with biopsy-proven nonalcoholic steatohepatitis classified as stage 1-3 fibrosis. Diabetes Metab Syndr Obes. 2018;11:835-843. eCollection 2018. doi:10.2147/DMSO.S184767

53. Yki-Jarvinen H. Thiazolidinediones. N Engl J Med. 2004;351:11061118. doi:10.1056/NEJMra041001

54. Ratziu V, Giral P, Jacqueminet S, et al.; LIDO Study Group. Rosiglitazone for nonalcoholic steatohepatitis: one-year results of the randomized placebo-controlled Fatty Liver Improvement with Rosiglitazone Therapy (FLIRT) Trial. Gastroenterology. 2008;135 (1):100-110. doi:10.1053/j.gastro.2008.03.078 Epub 2008 Apr 8.

55. Ratziu V, Charlotte F, Bernhardt C, et al.; LIDO Study Group. Longterm efficacy of rosiglitazone in nonalcoholic steatohepatitis: results of the fatty liver improvement by rosiglitazone therapy (FLIRT 2) extension trial. Hepatology. 2010;51(2):445-453. doi:10.1002/hep.23270

56. Belfort R, Harrison SA, Brown K, et al. A placebo-controlled trial of pioglitazone in subjects with nonalcoholic steatohepatitis. $N$ Engl J Med. 2006;355:2297-2307. doi:10.1056/NEJMoa060326

57. Aithal GP, Thomas JA, Kaye PV, et al. Randomized, placebocontrolled trial of pioglitazone in nondiabetic subjects with nonalcoholic steatohepatitis. J Gastroenterol. 2008;135(4):1176-1184. doi:10.1053/j.gastro.2008.06.047

58. Cusi K, Orsak B, Bril F, et al. Long-term pioglitazone treatment for patients with nonalcoholic steatohepatitis and prediabetes or type 2 diabetes mellitus: a randomized trial. Ann Intern Med. 2016;165 (5):305-315. doi:10.7326/M15-1774

59. Sanyal AJ, Chalasani N, Kowdley KV, et al. Pioglitazone, vitamin E, or placebo for nonalcoholic steatohepatitis. $N$ Engl J Med. 2010;362:1675-1685. doi:10.1056/NEJMoa0907929
60. Liao HW, Saver JL, Wu YL, et al. Pioglitazone and cardiovascular outcomes in patients with insulin resistance, pre-diabetes and type 2 diabetes: a systematic review and meta-analysis. BMJ Open. 2017; 7(1):e013927. doi:10.1136/bmjopen-2016-013927

61. Lincoff AM, Wolski K, Nicholls SJ, Nissen SE. Pioglitazone and risk of cardiovascular events in patients with type 2 diabetes mellitus: a meta-analysis of randomized trials. JAMA. 2007;298 (10):1180-1188. doi:10.1001/jama.298.10.1180

62. Baggio LL, Drucker DJ. Biology of incretins: GLP-1 and GIP. Gastroenterology. 2007;132:2131-2157. doi:10.1053/j.gastro. 2007.03.054

63. Deacon CF, Johnsen AH, Holst JJ. Degradation of glucagon-like peptide- 1 by human plasma in vitro yields an $\mathrm{N}$-terminally truncated peptide that is a major endogenous metabolite in vivo. J Clin Endocrinol Metab. 1995;80:952-957. doi:10.1210/jcem.80.3.7883856

64. Gupta NA, Mells J, Dunham RM, et al. Glucagon-like peptide-1 receptor is present on human hepatocytes and has a direct role in decreasing hepatic steatosis in vitro by modulating elements of the insulin signaling pathway. Hepatology. 2010;51(5):1584-1592. doi:10.1002/hep.23569

65. Wang XC, Gusdon AM, Liu H, Qu S. Effects of glucagon-like peptide-1 receptor agonists on non-alcoholic fatty liver disease and inflammation. World J Gastroenterol. 2014;20(40):14821-14830. doi:10.3748/wjg.v20.i40.14821

66. Bernsmeier C, Meyer-Gerspach AC, Blaser LS, et al. Glucoseinduced glucagon-like Peptide 1 secretion is deficient in patients with non-alcoholic fatty liver disease. PLoS One. 2014;9(1): e87488. doi:10.1371/journal.pone.0087488

67. Armstrong MJ, Gaunt P, Aithal GP, et al. Liraglutide safety and efficacy in patients with non-alcoholic steatohepatitis (LEAN): a multicentre, double-blind, randomised, placebo-controlled phase 2 study. Lancet. 2016;387(10019):679-690. doi:10.1016/S0140-6736 (15)00803-X

68. Feng W, Gao C, Bi Y, et al. Randomized trial comparing the effects of gliclazide, liraglutide, and metformin on diabetes with nonalcoholic fatty liver disease. $J$ Diabetes. 2017;9(8):800-809. doi:10.1111/1753-0407.12555

69. Shao N, Kuang HY, Hao M, Gao XY, Lin WJ, Zou W. Benefits of exenatide on obesity and non-alcoholic fatty liver disease with elevated liver enzymes in patients with type 2 diabetes. Diabetes Metab Res Rev. 2014;30(6):521-529. doi:10.1002/dmrr.2561

70. Fan H, Pan Q, Xu Y, Yang X. Exenatide improves type 2 diabetes concomitant with non-alcoholic fatty liver disease. Arq Bras Endocrinol Metabol. 2013;57(9):702-708. doi:10.1590/s000427302013000900005

71. ClinicalTrials.gov. Bethesda (MD): National Library of Medicine (US). $2000 \mathrm{Feb}$ 29. Identifier NCT02970942, Investigation of Efficacy and Safety of Three Dose Levels of Subcutaneous Semaglutide Once Daily Versus Placebo in Subjects With Non-alcoholic Steatohepatitis. Available from: https://clinicaltrials.gov/ct2/ show/NCT02970942?term $=$ NCT02970942\&draw=2\&rank=1. Accessed October 26, 2019.

72. Brigelius-Flohé R, Traber MG. Vitamin E: function and metabolism. Faseb J. 1999;13(10):1145-1155.

73. Parola M, Leonarduzzi G, Biasi F, et al. Vitamin E dietary supplementation protects against carbon tetrachloride-induced chronic liver damage and cirrhosis. Hepatology. 1992;16:1014-1021. doi:10.1002/hep.1840160426

74. Parola M, Muraca R, Dianzani I, et al. Vitamin E dietary supplementation inhibits transforming growth factor beta 1 gene expression in the rat liver. FEBS Lett. 1992;308:267-270. doi:10.1016/ 0014-5793(92)81290-3

75. Miller ER 3rd, Pastor-Barriuso R, Dalal D, Riemersma RA, Appel LJ, Guallar E. Meta-analysis: high-dosage vitamin E supplementation may increase all-cause mortality. Ann Intern Med. 2005;142(1):37-46. Epub 2004 Nov 10. doi:10.7326/0003-4819-142-1-200501040-00110 
76. Abner EL, Schmitt FA, Mendiondo MS, Marcum JL, Kryscio RJ. Vitamin E and all-cause mortality: a meta-analysis. Curr Aging Sci. 2011;4(2):158-170.

77. Schürks M, Glynn RJ, Rist PM, Tzourio C, Kurth T. Effects of vitamin $\mathrm{E}$ on stroke subtypes: meta-analysis of randomised controlled trials. BMJ. 2010;341:c5702. doi:10.1136/bmj.c5702

78. Klein EA, Thompson IM Jr, Tangen CM, et al. Vitamin E and the risk of prostate cancer: the Selenium and Vitamin E Cancer Prevention Trial (SELECT). JAMA. 2011;306(14):1549-1556. doi:10.1001/jama.2011.1437

79. Gaziano JM, Glynn RJ, Christen WG, et al. Vitamins E and C in the prevention of prostate and total cancer in men: the physicians' health study II randomized controlled trial. JAMA. 2009;301(1):5262. doi:10.1001/jama.2008.862 Epub 2008 Dec 9.

80. EASL-EASD-EASO Clinical Practice Guidelines for the management of non-alcoholic fatty liver disease. European Association for the Study of the Liver (EASL)., European Association for the Study of Diabetes (EASD)., European Association for the Study of Obesity (EASO). $J$ Hepatol. 2016;64(6):1388-1402. doi:10.1016/j.jhep.2015.11.004

81. Steinacher D, Claudel T, Trauner M. Therapeutic mechanisms of bile acids and nor-ursodeoxycholic acid in non-alcoholic fatty liver disease. Dig Dis. 2017;35(3):282-287. doi:10.1159/000454853

82. Xiang Z, Chen YP, Ma KF, et al. The role of ursodeoxycholic acid in non-alcoholic steatohepatitis: a systematic review. $B M C$ Gastroenterol. 2013;13:140.

83. Ratziu V, Ledinghen VD, Frederic O, et al. A multicentric, double-blind, randomized placebo-controlled trial of high dose ursodeoxycholic acid in patients with non-alcoholic steatohepatitis. Gastroenterology. 2009;136:P244. doi:10.1053/j.gastro.2008.11.049

84. Tilg H, Diehl AM. Cytokines in alcoholic and nonalcoholic steatohepatitis. N Engl J Med. 2000;343(20):1467-1476. doi:10.1056/ NEJM200011163432007

85. Schade UF. Pentoxifylline increases survival in murine endotoxin shock and decreases formation of tumor necrosis factor. Circ Shock. 1990;31:171-181.

86. Koppe SW, Sahai A, Malladi P, Whittington PF, Green RM. Pentoxifylline attenuates steatohepatitis induced by the methionine choline deficient diet. J Hepatol. 2004;41(4):592-598. doi:10.1016/ j.jhep.2004.06.030

87. Balibrea JL, Arias-Diaz J, Garcia C, Vara E. Effect of Pentoxifylline and somatostatin on tumour necrosis factor production by human pulmonary macrophages. Circ Shock. 1994;43(2):51-56.

88. Abdel Salam OM, Aiuomy AR, El-Shenawy SM, et al. Effect of pentoxifylline on hepatic injury caused in the rat by the administration of carbon tetrachloride or acetaminophen. Pharmacol Rep. 2005;57:596-603.

89. Zein CO, Lopez R, Fu X, et al. Pentoxifylline decreases oxidized lipid products in nonalcoholic steatohepatitis: new evidence on the potential therapeutic mechanism. Hepatology. 2012;56(4):12911299. doi: $10.1002 /$ hep. 25778

90. Satapathy SK, Garg S, Chauhan R, et al. Beneficial effects of tumor necrosis factor-alpha inhibition by pentoxifylline on clinical, biochemical, and metabolic parameters of patients with nonalcoholic steatohepatitis. Am J Gastroenterol. 2004;99:1946-1952. doi:10.1111/j.1572-0241.2004.40220.x

91. Adams LA, Zein CO, Angulo P, Lindor KD. A pilot trial of pentoxifylline in nonalcoholic steatohepatitis. Am J Gastroenterol. 2004;99:2365-2368. doi:10.1111/j.1572-0241.2004.40064.x

92. Van Wagner LB, Koppe SW, Brunt EM, et al. Pentoxifylline for the treatment of non-alcoholic steatohepatitis: a randomized controlled trial. Ann Hepatol. 2011;10(3):277-286.

93. Satapathy SK, Sakhuja P, Malhotra V, Sharma BC, Sarin SK. Beneficial effects of pentoxifylline on hepatic steatosis, fibrosis and necroinflammation in patients with non-alcoholic steatohepatitis. J Gastroenterol Hepatol. 2007;22:634-638. doi:10.1111/j.14401746.2006.04756.x
94. Zein CO, Yerian LM, Gogate P, et al. Pentoxifylline improves nonalcoholic steatohepatitis: a randomized placebo-controlled trial. Hepatology. 2011;54(5):1610-1619. doi:10.1002/hep.24544

95. Singh S, Khera R, Allen AM, Murad MH, Loomba R. Comparative effectiveness of pharmacological interventions for nonalcoholic steatohepatitis: A systematic review and network meta-analysis. Hepatology. 2015;62(5):1417-1432. doi:10.1002/hep.27999 Epub 2015 Oct 1.

96. Staels B, Rubenstrunk A, Noel B, et al. Hepatoprotective effects of the dual peroxisome proliferator-activated receptor alpha/delta agonist, GFT505, in rodent models of nonalcoholic fatty liver disease/ nonalcoholic steatohepatitis. Hepatology. 2013;58(6):1941-1952. doi:10.1002/hep.26461

97. Ratziu V, Harrison SA, Francque S, et al. Elafibranor, an agonist of the peroxisome proliferator-activated receptor- $\alpha$ and $-\delta$, induces resolution of nonalcoholic steatohepatitis without fibrosis worsening. Gastroenterology. 2016;150(5):1147-1159.e5. doi:10.1053/j. gastro.2016.01.038

98. ClinicalTrials.gov. Bethesda (MD): National Library of Medicine (US). 2000 Feb 29. Identifier NCT02704403, Phase 3 Study to Evaluate the Efficacy and Safety of Elafibranor Versus Placebo in Patients With Nonalcoholic Steatohepatitis (NASH) (RESOLVE-IT. Available from: https://clinicaltrials.gov/ct2/show/NCT02704403?term= NCT02704403\&draw=2\&rank=1. Accessed October 26, 2019.

99. Perelas A, Tsoulkani A, Perrea D. Effects of lipid-lowering drugs on adiponectin. Curr Vasc Pharmacol. 2010;8(6):836-848.

100. Dima A, Marinescu AG, Dima AC. Non-alcoholic fatty liver disease and the statins treatment. Rom J Intern Med. 2012;50(1):19-25.

101. Gómez-Domínguez E, Gisbert JP, Moreno-Monteagudo JA, GarcíaBuey L, Moreno-Otero R. A pilot study of atorvastatin treatment in dyslipemid, non-alcoholic fatty liver patients. Aliment Pharmacol Ther. 2006;23(11):1643-1647. doi:10.1111/j.1365-2036.2006.02926.x

102. Hyogo H, Tazuma S, Arihiro K, et al. Efficacy of atorvastatin for the treatment of nonalcoholic steatohepatitis with dyslipidemia. Metabolism. 2008;57(12):1711-1718. doi:10.1016/j.metabol. 2008.07.030

103. Foster T, Budoff MJ, Saab S, Ahmadi N, Gordon C, Guerci AD. Atorvastatin and antioxidants for the treatment of nonalcoholic fatty liver disease: the St Francis heart study randomized clinical trial. Am J Gastroenterol. 2011;106(1):71-77. doi:10.1038/ajg.2010.299

104. Ekstedt M, Franzén LE, Mathiesen UL, Holmqvist M, Bodemar G, Kechagias S. Statins in non-alcoholic fatty liver disease and chronically elevated liver enzymes: a histopathological follow-up study. $J$ Hepatol. 2007;47:135-141. doi:10.1016/j.jhep.2007.02.013

105. Kashani A, Phillips CO, Foody JM, et al. Risks associated with statin therapy. Circulation. 2006;114:2788-2797. doi:10.1161/ CIRCULATIONAHA.106.624890

106. Tziomalos K, Athyros VG, Paschos P, Karagiannis A. Nonalcoholic fatty liver disease and statins. Metabolism. 2015t;64 (10):1215-1223. doi:10.1016/j.metabol.2015.07.003

107. Ushio M, Nishio Y, Sekine O, et al. Ezetimibe prevents hepatic steatosis induced by a high-fat but not a high-fructose diet. Am J Physiol Endocrinol Metab. 2013;305(2):E293-304. doi:10.1152/ ajpendo.00442.2012

108. Nakade Y, Murotani K, Inoue T, et al. Ezetimibe for the treatment of non-alcoholic fatty liver disease: a meta-analysis. Hepatol Res. 2017;47(13):1417-1428. doi:10.1111/hepr.12887

109. Loomba R, Sirlin CB, Ang B, et al., San Diego Integrated NAFLD Research Consortium (SINC). Ezetimibe for the treatment of nonalcoholic steatohepatitis: assessment by novel magnetic resonance imaging and magnetic resonance elastography in a randomized trial (MOZART trial). Hepatology. 2015;61(4):1239-1250. doi:10.1002/hep.27647

110. Thomas C, Pellicciari R, Pruzanski M, Auwerx J, Schoonjans K. Targeting bile-acid signaling for metabolic diseases. Nat Rev Drug Discov. 2008;7:678-693. doi:10.1038/nrd2619 
111. Trauner M, Claudel T, Fickert P, Moustafa T, Wagner M. Bile acids as regulators of hepatic lipid and glucose metabolism. Dig Dis. 2010;28(1):220-224. doi:10.1159/000282091

112. Zhang Y, LaCerte C, Kansra S, Jackson JP, Brouwer KR, Edwards JE. Comparative potency of obeticholic acid and natural bile acids on FXR in hepatic and intestinal in vitro cell models. Pharmacol Res Perspect. 2017;5(6):e00368. doi:10.1002/prp2.368

113. Neuschwander-Tetri BA, Loomba R, Sanyal AJ, et al. Farnesoid X nuclear receptor ligand obeticholic acid for non-cirrhotic, non-alcoholic steatohepatitis (FLINT): a multicentre, randomised, placebocontrolled trial. Lancet. 2015;385(9972):956-965. doi:10.1016/ S0140-6736(14)61933-4

114. ClinicalTrials.gov. Bethesda (MD): National Library of Medicine (US). 2000 Feb 29. Identifier NCT02548351, Randomized Global Phase 3 Study to Evaluate the Impact on NASH With Fibrosis of Obeticholic Acid Treatment (REGENERATE). Available from: https://clinicaltrials. gov/ct2/show/NCT02548351?term=NCT02548351\&draw=2\&rank=1. Accessed October 26, 2019.

115. Li YS, Ni SY, Meng Y, et al. Angiotensin II facilitates fibrogenic effect of TGF- $\beta 1$ through enhancing the down-regulation of BAMBI caused by LPS: a new pro-fibrotic mechanism of angiotensin II. PLoS One. 2013;8(10):e76289. doi:10.1371/journal.pone.0076289

116. Shirai Y, Yoshiji H, Noguchi R, et al. Cross talk between toll-like receptor-4 signaling and angiotensin-II in liver fibrosis development in the rat model of non-alcoholic steatohepatitis. Gastroenterol Hepatol. 2013;28(4):723-730. doi:10.1111/jgh.12112

117. Yokohama S, Yoneda M, Haneda M. Therapeutic efficacy of an angiotensin II receptor antagonist in patients with nonalcoholic steatohepatitis. Hepatology. 2004;40:1222-1225. doi:10.1002/hep.20420

118. Georgescu EF, Georgescu M. Therapeutic options in non-alcoholic steatohepatitis (NASH). Are all agents all alike? Results of a preliminary study. J Gastrointest Liver Dis. 2007;16:39-46.

119. Torres DM, Jones FJ, Shaw JC, Williams CD, Ward JA, Harrison SA. Rosiglitazone versus rosiglitazone and metformin versus rosiglitazone and losartan in the treatment of nonalcoholic steatohepatitis in humans: a 12-month randomized, prospective, open- label trial. Hepatology. 2011;54(5):1631-1639. doi:10.1002/hep.24558

120. Georgescu EF, Ionescu R, Niculescu M, Mogoanta L, Vancica L. Angiotensin-receptor blockers as therapy for mild-to-moderate hypertension-associated non-alcoholic steatohepatitis. World $J$ Gastroenterol. 2009;15:942-954. doi:10.3748/wjg.15.942

121. Qiang G, Zhang L, Yang X, et al. Effect of valsartan on the pathological progression of hepatic fibrosis in rats with type 2 diabetes. Eur J Pharmacol. 2012;685(1-3):156-164. doi:10.1016/ j.ejphar.2012.04.028

122. Hong F, Chou HI, Friedman SL. Significant anti-fibrotic activity of cenicriviroc, a dual CCR2/CCR5 antagonist, in a rat model of thioacetamide-induced liver fibrosis and cirrhosis. Hepatology. 2013;58(6):1381A-1382A

123. Lefebvre E, Moyle G, Reshef R, et al. Antifibrotic effects of the dual CCR2/CCR5 antagonist cenicriviroc in animal models of liver and kidney fibrosis. PLoS One. 2016;11(6):e0158156. doi:10.1371/ journal.pone.0158156 eCollection 2016.

124. Seki E, de Minicis S, Inokuchi S, et al. CCR2 promotes hepatic fibrosis in mice. Hepatology. 2009;50(1):185-197. doi:10.1002/ hep. 22952

125. Friedman SL, Ratziu V, Harrison SA, et al. A randomized, placebocontrolled trial of cenicriviroc for treatment of nonalcoholic steatohepatitis with fibrosis. Hepatology. 2018;67(5):1754-1767. doi:10.1002/hep. 29477

126. ClinicalTrials.gov. Bethesda (MD): National Library of Medicine (US). 2000 Feb 29. Identifier NCT03028740, AURORA: Phase 3 Study for the Efficacy and Safety of CVC for the Treatment of Liver Fibrosis in Adults With NASH. Available from: https://clinicaltrials. gov/ct2/show/NCT03028740?term=NCT03028740\&draw $=$ 2\&rank=1. Accessed October 26, 2019.
127. Iruarrizaga-Lejarreta M, Varela-Rey M, Fernández-Ramos D, et al. Role of aramchol in steatohepatitis and fibrosis in mice. Hepatol Commun. 2017;1(9):911-927. doi:10.1002/hep4.1107

128. Ratziu V, de Guevara L, Safadi R, et al.; Sanyal AJ on behalf of the ARREST investigator study group. One-year results of the global phase $2 \mathrm{~b}$ randomized placebo controlled ARREST trial of aramchol, a stearoyl CoA desaturase modulator in NASH patients. Hepatology. 2018;68(Suppl1):LB-5.

129. ClinicalTrials.gov [Internet]. Bethesda (MD): National Library of Medicine (US). 2000 Feb 29. Identifier NCT04104321, A Phase 3/4 Clinical Study to Evaluate the Efficacy and Safety of Aramchol Versus Placebo in Subjects With NASH (ARMOR) (ARMOR). Sept 26, 2019 [cited 2019 Oct 26]. Available from: https://clinicaltrials.gov/ct2/show/ NCT04104321?term=aramchol\&draw=2\&rank=4

130. Loomba R, Lawitz E, Mantry PS, et al. The ASK1 inhibitor selonsertib in patients with nonalcoholic steatohepatitis: a randomized, phase 2 trial [published online ahead of print, 2017 Dec 26] [published correction appears in Hepatology. 2018 May;67(5):2063]. Hepatology. 2017;67 (2):549-559. doi:10.1002/hep.29514

131. ClinicalTrials.gov. Bethesda (MD): National Library of Medicine (US). 2000 Feb 29. Identifier NCT03053050, Safety and Efficacy of Selonsertib in Adults With Nonalcoholic Steatohepatitis (NASH) and Bridging (F3) Fibrosis (STELLAR 3). Available from: https://clinical trials.gov/ct $2 /$ show $/$ NCT03053050?term $=$ NCT03053050\&draw $=$ 2\&rank=1. Accessed October 26, 2019.

132. ClinicalTrials.gov. Bethesda (MD): National Library of Medicine (US). 2000 Feb 29. Identifier NCT03053063, Safety and Efficacy of Selonsertib in Adults With Compensated Cirrhosis Due to Nonalcoholic Steatohepatitis (NASH) (STELLAR 4). Available from: https://clinicaltrials.gov/ct2/ show/NCT03053063?term=NCT03053063\&draw=2\&rank=1. Accessed October 26, 2019.

133. Loomba R, Kayali Z, Noureddin M, et al. GS-0976 reduces hepatic steatosis and fibrosis markers in patients with nonalcoholic fatty liver disease. Gastroenterology. 2018;155(5):1463-1473. doi:10.1053/j. gastro.2018.07.027

134. Luo Y, Krupinski J, Gao S, Charles ED, Christian R. BMS986036, a PEGylated fibroblast growth factor 21 analogue, reduces fibrosis and PRO-C3 in a mouse model of non-alcoholic steatohepatitis. J Hepatol. 2018;68:S396-S397. doi:10.1016/ S0168-8278(18)31028-6

135. Sanyal A, Charles ED, Neuschwander-Tetri BA, et al. Pegbelfermin (BMS-986036), a PEGylated fibroblast growth factor 21 analogue, in patients with non-alcoholic steatohepatitis: a randomised, double-blind, placebo-controlled, phase 2a trial. Lancet. 2019;392 (10165):2705-2717. doi:10.1016/S0140-6736(18)31785-9

136. Loomba R, Neutel J, Mohseni R, et al. VK2809, a novel liverdirected thyroid receptor beta agonist, significantly reduces liver fat with both low and high doses in patients with nonalcoholic fatty liver disease: a phase 2 randomized, placebocontrolled trial. J Hepatol. 2019;70:e150-151. doi:10.1016/ S0618-8278(19)30266-X

137. ClinicalTrials.gov. Bethesda (MD): National Library of Medicine (US). 2000 Feb 29. Identifier NCT02442687, JKB-121 for the Treatment of Nonalcoholic Steatohepatitis. Available from: https://clinicaltrials.gov/ ct2/show/results/NCT02442687?term $=$ NCT02442687\&draw $=$ 2\&rank=1. Accessed October 26, 2019.

138. Harrison SA, Abdelmalek MF, Caldwell S, et al. Simtuzumab is ineffective for patients with bridging fibrosis or compensated cirrhosis caused by nonalcoholic steatohepatitis. Gastroenterology. 2018;155(4):1440-1453. doi:10.1053/j.gastro.2018.07.006

139. ClinicalTrials.gov. Bethesda (MD): National Library of Medicine (US). 2000 Feb 29. Identifier NCT01672879, Simtuzumab (SIM, GS-6624) in the Treatment of Cirrhosis Due to NASH (NASH). Available from: https:/clinicaltrials.gov/ct2/show/results/NCT01672879?term= NCT01672879\&draw=2\&rank=1. Accessed October 26, 2019. 
140. ClinicalTrials.gov. Bethesda (MD): National Library of Medicine (US). 2000 Feb 29. Identifier NCT01672866, Safety and Efficacy of Simtuzumab (SIM, GS-6624) in Adults With Advanced Liver Fibrosis But Not Cirrhosis Secondary to Non-Alcoholic Steatohepatitis (NASH). Available from: https://clinicaltrials.gov/ ct $2 /$ show $/$ results $/$ NCT01672866? cond $=$ NCT01672866\&draw $=$ 2\&rank=1. Accessed October 26, 2019.

141. ClinicalTrials.gov. Bethesda (MD): National Library of Medicine (US). 2000 Feb 29. Identifier NCT02686762, Emricasan, a Caspase Inhibitor, for Evaluation in Subjects With Non-Alcoholic Steatohepatitis (NASH) Fibrosis. Available from: https:/clinical trials.gov/ct $2 /$ results? cond $=\&$ term $=+$ NCT02686762\& cntry $=$ \&state $=\&$ city $=\&$ dist $=$. Accessed October 26, 2019.

142. ClinicalTrials.gov. Bethesda (MD): National Library of Medicine (US). 2000 Feb 29. Identifier NCT02462967, Clinical Trial to Evaluation the Safety and Efficacy of GRMD-02 for the Treatment of Liver Fibrosis and Resultant Portal Hypertension in Patients With Nash Cirrhosis (NASHCX). Available from: https://clinicaltrials.gov/ct2/show/ NCT02462967?term=NCT02462967\&draw=2\&rank=1. Accessed October 26, 2019.

143. Chalasani N, Garcia-Tsao G, Goodman Z, et al. A multicenter, randomized, double-blind, PLB-controlled trial of Galectin-3 inhibitor (GR-MD-02) in patients with NASH cirrhosis and portal hypertension. J Hepatol. 2018;68(1):S100-01. doi:10.1016/S01688278(18)30420-3
144. Harrison S, Moussa S, Bashir M, et al. MGL-3196, a selective thyroid hormone receptor-beta agonist significantly decreases hepatic fat in NASH patients at 12 weeks, the primary endpoint in a 36 week serial liver biopsy study. $J$ Hepatol. 2018;68:S38. doi:10.1016/S0168-8278(18)30292-7

145. Patel K, Harrison SA, Trotter JF, et al. The non-steroidal FXR agonist GS-9674 leads to significant reductions in hepatic steatosis, serum bile acids, and liver biochemistry in a phase 2, randomized, placebo-controlled trial of patients with NASH. Hepatology. 2019;70(S1):438A.

146. Harrison SA, Rinella ME, Abdelmalek MF, et al. NGM282 for treatment of non-alcoholic steatohepatitis: a multicentre, randomised, double-blind, placebo-controlled, phase 2 trial. Lancet. 2018;391(10126):1174-1185. doi:10.1016/S0140-6736(18)30474-4

147. Chalasani N, Younossi Z, Lavine JE, et al. The diagnosis and management of nonalcoholic fatty liver disease: Practice guidance from the American Association for the Study of Liver Diseases. Hepatology. 2018;67(1):328-357. doi:10.1002/hep.29367.

148. Tahara A, Kurosaki E, Yokono M, et al. Effects of SGLT2 selective inhibitor ipragliflozin on hyperglycemia, hyperlipidemia, hepatic steatosis, oxidative stress, inflammation, and obesity in type 2 diabetic mice. Eur J Pharmacol. 2013 Sep 5;715(1-3):246-55. doi:10.1016/j.ejphar.2013.05.014.

149. Qiang S, Nakatsu Y, Seno Y, et al. Treatment with the SGLT2 inhibitor luseogliflozin improves nonalcoholic steatohepatitis in a rodent model with diabetes mellitus. Diabetol Metab Syndr. 2015;7:104pmid:26594248

\section{Publish your work in this journal}

Hepatic Medicine: Evidence and Research is an international, peerreviewed, open access journal covering all aspects of adult and pediatric hepatology in the clinic and laboratory including the following topics: Pathology, pathophysiology of hepatic disease; Investigation and treatment of hepatic disease; Pharmacology of drugs used for the treatment of hepatic disease. Issues of patient safety and quality of care will also be considered. The manuscript management system is completely online and includes a very quick and fair peer-review system, which is all easy to use. Visit http://www.dovepress.com/ testimonials.php to read real quotes from published authors. 